\title{
Metabolic alterations in the erythrocyte during blood-stage development of the malaria parasite
}

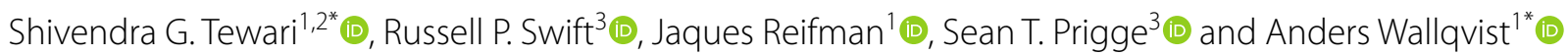

\begin{abstract}
Background: Human blood cells (erythrocytes) serve as hosts for the malaria parasite Plasmodium falciparum during its 48-h intraerythrocytic developmental cycle (IDC). Established in vitro protocols allow for the study of host-parasite interactions during this phase and, in particular, high-resolution metabolomics can provide a window into host-parasite interactions that support parasite development.
\end{abstract}

Methods: Uninfected and parasite-infected erythrocyte cultures were maintained at $2 \%$ haematocrit for the duration of the IDC, while parasitaemia was maintained at $7 \%$ in the infected cultures. The parasite-infected cultures were synchronized to obtain stage-dependent information of parasite development during the IDC. Samples were collected in quadruplicate at six time points from the uninfected and parasite-infected cultures and global metabolomics was used to analyse cell fractions of these cultures.

Results: In uninfected and parasite-infected cultures during the IDC, 501 intracellular metabolites, including 223 lipid metabolites, were successfully quantified. Of these, 19 distinct metabolites were present only in the parasite-infected culture, 10 of which increased to twofold in abundance during the IDC. This work quantified approximately five times the metabolites measured in previous studies of similar research scope, which allowed for more detailed analyses. Enrichment in lipid metabolism pathways exhibited a time-dependent association with different classes of lipids during the IDC. Specifically, enrichment occurred in sphingolipids at the earlier stages, and subsequently in lysophospholipid and phospholipid metabolites at the intermediate and end stages of the IDC, respectively. In addition, there was an accumulation of 18-, 20-, and 22-carbon polyunsaturated fatty acids, which produce eicosanoids and promote gametocytogenesis in infected erythrocyte cultures.

Conclusions: The current study revealed a number of heretofore unidentified metabolic components of the hostparasite system, which the parasite may exploit in a time-dependent manner to grow over the course of its development in the blood stage. Notably, the analyses identified components, such as precursors of immunomodulatory molecules, stage-dependent lipid dynamics, and metabolites, unique to parasite-infected cultures. These conclusions are reinforced by the metabolic alterations that were characterized during the IDC, which were in close agreement with those known from previous studies of blood-stage infection.

\footnotetext{
*Correspondence: stewari@bhsai.org; sven.a.wallqvist.civ@mail.mil

1 Department of Defense Biotechnology High Performance Computing

Software Applications Institute, Telemedicine and Advanced Technology

Research Center, U.S. Army Medical Research and Development

Command, Ft. Detrick, MD, USA

Full list of author information is available at the end of the article
}

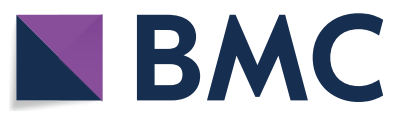

(c) The Author(s) 2020. This article is licensed under a Creative Commons Attribution 4.0 International License, which permits use, sharing, adaptation, distribution and reproduction in any medium or format, as long as you give appropriate credit to the original author(s) and the source, provide a link to the Creative Commons licence, and indicate if changes were made. The images or other third party material in this article are included in the article's Creative Commons licence, unless indicated otherwise in a credit line to the material. If material is not included in the article's Creative Commons licence and your intended use is not permitted by statutory regulation or exceeds the permitted use, you will need to obtain permission directly from the copyright holder. To view a copy of this licence, visit http://creativeco mmons.org/licenses/by/4.0/. The Creative Commons Public Domain Dedication waiver (http://creativecommons.org/publicdomain/ zero/1.0/) applies to the data made available in this article, unless otherwise stated in a credit line to the data. 
Keywords: Plasmodium falciparum, Host-parasite metabolism, Blood-stage infection, Metabolome, Lysophosphatidylglycerol, Polyunsaturated fatty acids

\section{Background}

In 2017, there were 219 million cases of malaria worldwide and the ten most burdened African countries saw an estimated 3.5 million more cases than in 2016 [1]. Plasmodium falciparum is responsible for $99.7 \%$ of all malaria cases in the World Health Organization (WHO) African region, which accounted for $93 \%$ of all malarial deaths in 2017 [1]. During the symptomatic stage of malaria, $P$. falciparum resides in human blood cells (erythrocytes) as it multiplies asexually during the 48-h intraerythrocytic developmental cycle (IDC) [2]. The human erythrocyte is also the main conduit for providing $P$. falciparum with essential nutrients during its development during the IDC [3]. While the interactions of the parasite with its host, the human erythrocyte, have been studied for well over a century, much remains to be characterized and discovered. For example, although parasiteinfected erythrocytes rapidly sequester arginine from the culture medium under in vitro conditions [4], the relevance of this to parasite development is unclear. In recent years, high-resolution metabolomic methods have been employed to improve the understanding of host-parasite interactions, with the aim of ultimately identifying novel treatments and diagnostic strategies [5-7].

Here, synchronous cultures of the $P$. falciparum parasite were generated in human erythrocytes and globally targeted mass spectrometry was employed to quantify metabolic changes in uninfected and parasite-infected erythrocyte cultures during the IDC. Specifically, the aim of the study was to characterize parasite development during this phase at six equally spaced time points that roughly covered its early, intermediate, and late stages. Although previous studies $[4,8]$ have also examined metabolomic data at several time points, their methods primarily quantified metabolites involved in carbohydrate, amino acid, and nucleotide metabolism. Yet, during the IDC, $P$. falciparum also synthesizes lipids that are essential not only for membrane biogenesis but also for lipid-dependent signaling or trafficking processes [9]. Therefore, extraction and detection methods that reproducibly quantify $\sim 850$ metabolites (of which roughly half are lipids) across different cohorts and disease conditions were employed to track these lipids.

To delineate $P$. falciparum metabolism using metabolomic data collected during the IDC, a series of computational methods were used and key global, pathway-level, and stage-specific metabolites were identified. This revealed time-dependent and time-independent alterations in nucleotide, lipid, and carbohydrate metabolites, which were associated with parasite development. Analyses of uninfected and parasite-infected cultures showed significant enrichment in metabolites associated with lipid synthesis. Specifically, lipids showed significant enrichment, which was associated with increased abundance of several polyunsaturated fatty acids (PUFAs) in infected cultures relative to uninfected cultures. Stagedependent analyses revealed dynamic alterations in the abundance of lysophosphatidylglycerol (LPG) metabolites. The results provide an initial explanation of how LPG metabolites could contribute to P. falciparum development during the IDC.

\section{Methods \\ Parasite culture, purification of erythrocytes, and sample collection}

Plasmodium falciparum NF54 parasites (generously provided by David Fidock, Columbia University) were propagated in O-positive human erythrocytes at 2\% haematocrit in gassed flasks $\left(94 \% \mathrm{~N}_{2}, 3 \% \mathrm{O}_{2}\right.$, and $3 \%$ $\left.\mathrm{CO}_{2}\right)$ at $37^{\circ} \mathrm{C}$. Human erythrocytes were obtained as part of an institutional review board-approved phlebotomy protocol (NA_00019050) and used within 2 days after isolation. The infected erythrocytes were maintained in Roswell Park Memorial Institute (RPMI) 1640 medium (Gibco, Gaithersburg, MD) and supplemented with $20 \mathrm{mM}$ HEPES, $12.5 \mu \mathrm{g} / \mathrm{mL}$ hypoxanthine, $0.3 \%$ sodium bicarbonate, $25 \mu \mathrm{g} / \mathrm{mL}$ gentamicin, $0.5 \mu \mathrm{M}$ R-lipoic acid, and $0.5 \%$ AlbuMAX II (Life Technologies Inc., Carlsbad, CA). Erythrocytes depleted of white blood cells (WBCs) were used for the parasite culture. First, the buffy coat was removed following two rounds of density gradient centrifugation. The enriched erythrocytes were then overlaid on a $60 \%$ Percoll solution and centrifuged at $1500 \times g$ for $30 \mathrm{~min}$. After removal of WBCs from the interface, the pelleted erythrocytes were carefully collected and washed several times in RPMI-1640 before the haematocrit was adjusted to $50 \%$.

To generate synchronized parasites, the cultures were passed through magnetically activated cell sorting (MACS) columns (Miltenyi Biotec, Auburn, CA) and purified, every $44-48 \mathrm{~h}$ for 4 days before the initiation of the experiment. Giemsa-stained blood smears and light microscopy were used to monitor parasitaemia and synchronicity. Additionally, immediately before sample collection, the absence of contaminating mycoplasma was confirmed by a polymerase chain reaction, using primers 
specific for the gene encoding $16 \mathrm{~S}$ ribosomal RNA (5'GGAGCAAACAGGATTAGATACCC and 5'-CACCAT CTGTCACTCTGTTAACC).

Before data collection, a synchronized parasite culture $(300 \mathrm{~mL})$ was passed through a MACS column in four $75-\mathrm{mL}$ volumes, each eluted with $20 \mathrm{~mL}$ of culture medium. The eluates were pooled and adjusted to a total culture volume of $300 \mathrm{~mL}$ at $2 \%$ haematocrit using leukodepleted blood, and then the culture was divided into four $75-\mathrm{mL}$ replicate flasks. Four flasks containing $50 \mathrm{~mL}$ of media with uninfected leukodepleted erythrocytes at $2 \%$ haematocrit provided the control group samples for the metabolomic analysis. MACS purification resulted in elution of late-stage trophozoites. The cultures were regularly observed via blood smear until $0-2 \mathrm{~h}$ after merozoite invasion of the erythrocytes (final parasitaemia of 7\%), at which point the culture media in all flasks was replaced with fresh media (time 0 for this experiment).

Cells were harvested from the four test flasks and the four control flasks via collection of at least $7 \mathrm{~mL}$ of culture from each flask. Then, after centrifugation of the tubes at $1500 \times g$ for $5 \mathrm{~min}$ to pellet the cells, followed by aspiration of the media, $100 \mu \mathrm{L}$ of the test- or control-cell pellets was transferred to $1.5-\mathrm{mL}$ tubes, which were flash frozen in an ethanol/dry-ice bath and stored at $-80{ }^{\circ} \mathrm{C}$ for subsequent metabolomic analysis. This procedure was repeated at the following times during the IDC: 0,8 , 16, 24, 32, and $40 \mathrm{~h}$. Finally, quadruplicate samples were sent to Metabolon, Inc. (Durham, NC) for metabolite analysis.

\section{Global metabolomic profiling of intracellular data}

Staff at Metabolon Inc. inventoried the samples and then immediately stored them at $-80{ }^{\circ} \mathrm{C}$. The protocol for metabolomic profiling involved the following steps: (1) precipitate the proteins with methanol under vigorous shaking for 2 min using a GenoGrinder 2000 (Glen Mills Inc., Clifton, NJ), (2) centrifuge the precipitate to remove the proteins, dissociate small molecules bound to the proteins or trapped in the precipitated protein matrix, and recover chemically diverse metabolites, and (3) divide the resulting extract into five fractions [two for analysis by two separate reverse-phase (RP) ultrahighperformance liquid chromatography (UPLC) tandem mass spectrometry (MS/MS) methods with positive ion mode electrospray ionization (ESI), one for analysis by RP/UPLC-MS/MS with negative ion mode ESI, one for analysis by hydrophilic-interaction chromatography (HILIC) UPLC-MS/MS with negative ion mode ESI, and one for use as a backup sample]. All methods involved the use of a Waters ACQUITY UPLC system (Waters Corp., Milford, MA) and a Q-Exactive high resolution/accurate mass spectrometer (Thermo Fisher Scientific, Hampton,
$\mathrm{NH}$ ) interfaced with a heated electrospray ionization (HESI-II) source and Orbitrap mass analyser operated at 35,000 mass resolution.

Based on the profiling results provided by Metabolon Inc., a total of 501 metabolites of known identity (designated metabolites in the Metabolon $\odot$ library) were quantified. The raw data were normalized by the Bradford protein concentration of each sample and then any missing value of a metabolite was imputed with its minimum observed value across all samples.

\section{Global analysis of the data}

The intracellular metabolomic data from uninfected ( $u R B C)$ and parasite-infected (iRBC) cultures were used as input to the 'clustergram' function built into MATLAB $^{\circledR}$. Ward's hierarchical clustering method and the Euclidean distance metric were used to cluster metabolites with similar temporal profiles in the uRBC and iRBC cultures. Quantile normalization was performed on the filtered data before visualizing the data in the form of a heat map. Principal component analysis (PCA) was performed on the entire data set to identify any separation between the $\mathrm{uRBC}$ and $\mathrm{iRBC}$ cultures along three principal axes. This was achieved with the use of the 'pca' function built into MATLAB ${ }^{\circledR}$.

Fisher's exact test was used to determine whether a cluster was enriched in a specific metabolite class. Specifically, the following contingency table was computed for a given cluster:

$$
\left[\begin{array}{ll}
\mathrm{N}_{\mathrm{c}} & \mathrm{N}_{\mathrm{c}}^{\prime} \\
\mathrm{N}_{\mathrm{d}} & \mathrm{N}_{\mathrm{d}}^{\prime}
\end{array}\right]
$$

where $\mathrm{N}_{\mathrm{c}}$ and $\mathrm{N}_{\mathrm{d}}$ denote the number of metabolites that belong to metabolite class ' $\mathrm{K}$ ' in cluster ' $\mathrm{C}$ ' and in the entire data set, respectively, and $\mathrm{N}_{\mathrm{c}}^{\prime}$ and $\mathrm{N}_{\mathrm{d}}^{\prime}$ represent the number of metabolites that do not belong to class $\mathrm{K}$ in cluster $C$ and in the entire data set, respectively. The 'fishertest' function built into MATLAB ${ }^{\circledR}$ was used to test the null hypothesis that there was no nonrandom association between metabolite class $\mathrm{K}$ and cluster $\mathrm{C}$. If the test rejected the null hypothesis at the 5\% significance level, cluster $\mathrm{C}$ was deemed to be enriched in metabolite class K.

The average fold change in metabolites detected in the $\mathrm{uRBC}$ and $\mathrm{iRBC}$ cultures was computed to identify metabolites that were significantly altered during the IDC. To this end, the 'bootstrp' function built into MATLAB $^{\circledR}$ was used to generate 10,000 bootstrap samples for each metabolite from four replicate measurements of abundance at each time point. The fold change was then computed according to the following equation: 


$$
\mathrm{F}_{\mathrm{i}}=\frac{\overline{\mathrm{m}}_{\mathrm{iRBC}}}{\overline{\mathrm{m}}_{\mathrm{uRBC}}}
$$

where $F_{i}$ denotes the fold change in a metabolite ' $m$ ' for the ith bootstrap sample, and $\overline{\mathrm{m}}_{\mathrm{iRBC}}$ and $\overline{\mathrm{m}}_{\mathrm{uRBC}}$ represent the average abundance levels of the metabolite in the iRBC and $\mathrm{uRBC}$ cultures, respectively, where abundance is averaged across all replicates and time points. To obtain the average and standard deviation of the fold change in a metabolite during the IDC, the average and standard deviation of $F_{i}$ were computed across all samples. To obtain the average fold change of a metabolite at a given time point, the same procedure was used, but with $F_{i}$ from the abundance measurements for that time point.

\section{Pathway enrichment and statistical analyses}

To gain mechanistic insights into the altered metabolic pathways, quantitative pathway enrichment analysis (QSEA) was performed using MetaboAnalyst [10] on the processed intracellular metabolomic data from uRBC and iRBC cultures. QSEA identifies associations between metabolite sets and disease conditions, such as parasite infection. The MetaboAnalyst [10] Web tool requires Human Metabolome Database (HMDB) identifiers as input to perform QSEA. Metabolite sets related to human metabolism, which contained at least five metabolites per set, were obtained from the small molecule pathway database (SMPDB). Starting with the SMPDB library [11] of normal human metabolic pathways, KEGG pathway annotations [12] were used to further classify the SMPDB metabolic pathways into six major pathways. Before the analysis, the data were grouped into three different IDC periods, i.e., $0-8,16-24$, and 32-40 h, to characterize stage-relevant parasite development and to increase the sample size for detecting statistically significant differences within each group [13].

To test for infection-specific alterations in metabolites, for each of the 501 metabolites, a two-way analysis of variance (ANOVA) was performed for each IDC stage (early, intermediate, and late), with time point ( 0 and $8 \mathrm{~h}$, 16 and $24 \mathrm{~h}$, or 32 and $40 \mathrm{~h}$ ) and infection status (infected or uninfected) as the between-group factors. Prior to the ANOVA, the data were log (base 2)-transformed to make them normally distributed [14]. The 'anova2' function built into MATLAB was used to analyse abundance levels for each metabolite to test the degree to which they differed as a function of the IDC period, infection status, and their interaction, at a significance criterion of $p \leq 0.05$. At each of the three IDC stages, a metabolite that showed a significant interaction between time point and infection status was deemed as an infection-specific metabolite, because this term would capture any change in the difference in the abundance of such a metabolite between the iRBC and $\mathrm{uRBC}$ cultures over time. Lastly, the 'mafdr' function built into MATLAB was used to implement Storey's method [15] to estimate the false discovery rate for multiple hypothesis testing. A significantly altered metabolite was rejected if the false discovery rate was $10 \%$ or greater (i.e., $q \geq 0.10$ ).

\section{Results \\ Metabolomics of blood-stage malaria parasites}

Recent years have seen a surge in the use of metabolomics to probe biological and physiological systems of interest. Specifically, methods have been developed to quench the metabolism of parasite-infected erythrocytes and study their metabolite extracts [6]. Metabolic profiling approaches are used to characterize blood-stage parasite development [4, 16], discover biomarkers [17, 18], and identify novel therapeutic targets [19]. Table 1 lists studies that have examined the blood stage of malaria parasites using metabolomics. Of note are two studies $[4,8]$ that characterized more than 100 metabolites in synchronous cultures of $P$. falciparum at several time points during the IDC. Figure 1 shows a comparison of the metabolite coverage for these studies and the current study, as well as the overlap of metabolites between the studies. Whereas the number of nucleotide metabolites was comparable across the three studies, that of the lipid metabolites was many-fold higher in the current study (Fig. 1b). The extraction methods and mass spectrometry platforms used in previous studies precluded them from detecting a large number of lipid metabolites $[4,8]$. Several recent studies $[6,23,24]$ quantified more metabolites than in the studies by Babbitt et al. [8] or Olszewski et al. [4]. However, they either did not examine all parasite stages or did not include matched uninfected erythrocyte cultures during the IDC (see Table 1). Therefore, it was not possible to comprehensively compare the current study with the recent studies. In the following sections, a global analysis of the collected data will be presented first. Subsequently, the metabolic changes that characterize parasite development during the IDC will be examined.

\section{Global metabolomic profiling of $\mathrm{URBC}$ and iRBC cultures}

Five hundred and one metabolites present in either $\mathrm{uRBC}$ or iRBC cultures were quantified. Of these, 481 were present in both cultures and 20 were unique to iRBC cultures. An analysis of the former set is presented first, followed by an analysis of the iRBC-specific metabolites in the subsection titled "Metabolites uniquely associated with parasite infection" of the paper. Of the 501 metabolites, 333 and 261 were annotated in the HMDB [25] and Plasmodium [26] metabolite databases, respectively, with 9 unique to the latter. A total of 159 metabolites were 
Table 1 Metabolomic data obtained from blood-stage parasites

\begin{tabular}{|c|c|c|c|c|c|}
\hline $\mathrm{N}_{\text {metabolites }}$ & $\mathrm{N}_{\text {time }}$ & $n^{\mathrm{a}}$ & Source of data & Year & References \\
\hline \multicolumn{6}{|c|}{ Obtained at multiple time points from uninfected and infected erythrocytes } \\
\hline 104 & 7 & 3 & Uninfected and parasite-infected synchronous cultures of erythrocytes & 2009 & {$[4]$} \\
\hline 120 & 10 & 2 & Uninfected and parasite-infected synchronous cultures of erythrocytes & 2012 & {$[8]$} \\
\hline 501 & 6 & 4 & Uninfected and parasite-infected synchronous cultures of erythrocytes & - & This study \\
\hline \multicolumn{6}{|c|}{ Other relevant data obtained during the IDC } \\
\hline 52 & 1 & 13 & Saponin-treated synchronous parasite culture & 2009 & {$[16]$} \\
\hline 104 & 1 & 4 & $\begin{array}{l}\text { Uninfected and parasite-infected asynchronous cultures of erythrocytes } \\
\text { (absence/presence of streptolysin O) }\end{array}$ & 2013 & {$[20]$} \\
\hline 76 & $5^{b}$ & 6 & Parasite-infected synchronous cultures of erythrocytes & 2016 & {$[21]$} \\
\hline 113 & 1 & 3 & Parasite-infected synchronous cultures of erythrocytes & 2016 & {$[22]$} \\
\hline 460 & 1 & 4 & Parasite-infected synchronous cultures of erythrocytes & 2016 & {$[6]$} \\
\hline 583 & 1 & $3^{c}$ & Uninfected and parasite-infected synchronous cultures of erythrocytes & 2017 & {$[23]$} \\
\hline 297 & 1 & 5 & Saponin-treated synchronous parasite culture & 2018 & {$[24]$} \\
\hline
\end{tabular}

$N_{\text {metabolites }}$ number of metabolites quantified, $N_{\text {time }}$ number of time points sampled during the intraerythrocytic developmental cycle (IDC)

a Number of replicates at each time point

b Time points cover the first $6 \mathrm{~h}$ after treatment by a drug

c Data from uninfected erythrocytes do not have a replicate
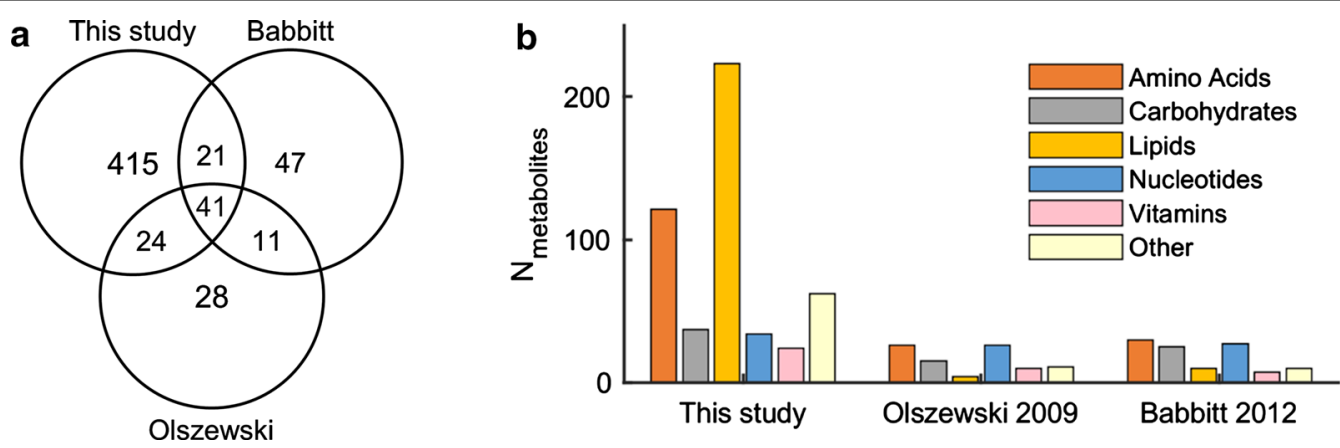

Fig. 1 Comparison of metabolite coverage across this study and studies by Babbitt et al. [8] and Olszewski et al. [4]. a Venn diagram showing overlap of metabolites between the three studies. Relative to the other two studies, which quantified comparable numbers of metabolites during the intraerythrocytic developmental cycle (IDC), this study quantified roughly five times more metabolites. $\mathbf{b}$ Metabolites detected in the three studies ( $\mathrm{N}_{\text {metabolites }}$ ) mapped onto five major metabolic pathways. In contrast to the previous studies, which quantified $\sim 100$ metabolites during the IDC, this study quantified over 200 lipid metabolites and more than 100 amino acid metabolites. "Other" denotes metabolites that do not belong to the five major metabolic pathways

not annotated in either database, although it was possible to assign 2 of them to KEGG identifiers. Thirty-one percent $(157 / 501)$ of the metabolites could not be linked back to any of these databases (Additional file 1), indicating a general lack of specific pathway information for these compounds. Of these unannotated metabolites, $79 \%(124 / 157)$ and $8 \%(12 / 157)$ belonged to pathways involved in lipid metabolism and amino acid metabolism, respectively. A small percentage (7/157) of the unannotated metabolites belonged to pathways involved in xenobiotic metabolism. Additional files 2 and 3 include lists of all of the metabolites found in the Plasmodium metabolite database [26] and Malaria Parasite Metabolic Pathways database [27], respectively.

Figure 2a shows the individual $\log _{2}$ fold-change values for all identified metabolites as measured for each replicate at each time point. Compared to iRBC cultures, which showed increases in fold-change values late in the IDC (32-40 h), uRBC cultures showed fewer metabolite changes over time. To ascertain the overall characteristics and time-dependent alterations of the metabolite data, PCA was performed on the fold-change data in Fig. 2a. Figure 2b shows a clear separation of the data for the two culture systems over time, with the four replicate 


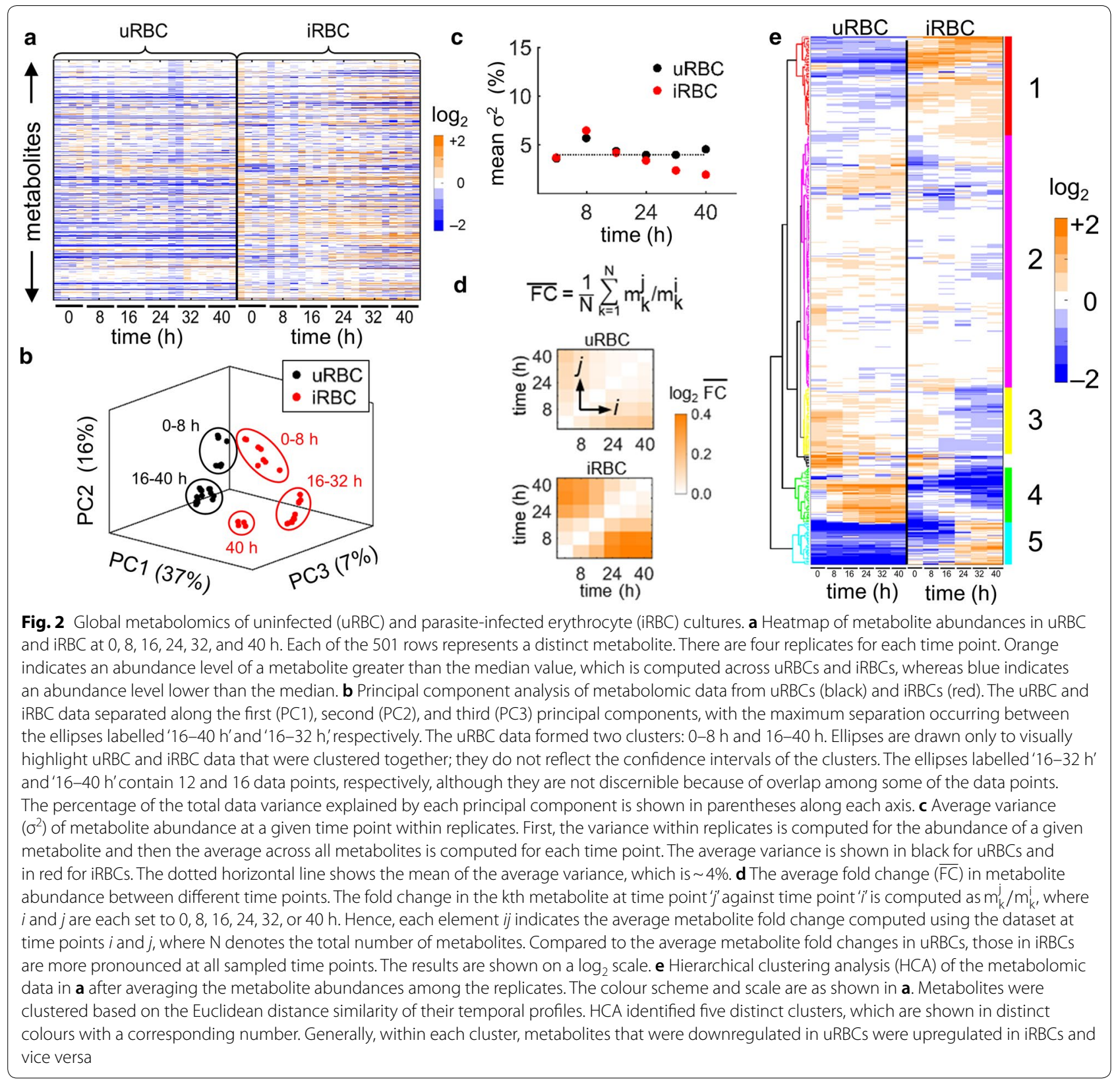

data points for each time point allowing visualization and assessment of the data spread.

Five ellipses visually separated the data into five clusters $(0-8 \mathrm{~h}, 0-8 \mathrm{~h}, 16-32 \mathrm{~h}, 16-40 \mathrm{~h}$, and $40 \mathrm{~h})$, each of which contains either $\mathrm{URBC}$ or iRBC data plotted along the three principal components for the indicated time range. Not all of the replicates are discernible because some data points overlap. For example, the ellipses labelled ' $16-32 \mathrm{~h}$ ' and ' $16-40 \mathrm{~h}$ ' contain 12 and 16 data points, respectively. Early in the IDC $(0-8 \mathrm{~h})$, the data for the two sets of cultures (infected and uninfected) were not identical but similar. It should be noted that the uninfected cultures and the cultures infected with synchronized late-stage parasites were incubated for several hours until ring-stage parasites were observed and sample collection began $(t=0)$. This incubation period and the relatively low metabolic activity of the new ring-stage parasites are likely responsible for the slight separation between $\mathrm{URBC}$ and iRBC cultures along the first principal component $(\mathrm{PC} 1)$ at $0-8 \mathrm{~h}$. The uRBC cultures underwent metabolic changes over time, as shown by the separation along the second principal 
component (PC2) between the $0-8 \mathrm{~h}$ and $16-40 \mathrm{~h}$ time points. The separation between the $\mathrm{uRBC}$ and $\mathrm{iRBC}$ data along PC1 was greatest late in the IDC (16-32 h), coinciding with the appearance of trophozoites and schizonts in iRBCs. At the last data point $(40 \mathrm{~h})$, there was less separation between $u R B C$ and iRBC cultures. That is, the metabolic profile of iRBC cultures corresponding to the late-stage schizogony of the parasite began to resemble that of uRBC cultures at 16-40 h. To ascertain measurement variation, the variance in the data arising from replicate measurements was calculated for both culture systems. Figure $2 \mathrm{c}$ shows the average metabolite variance for uRBCs and iRBCs as a function of time of data collection. For both systems, the average relative variance was $\sim 4 \%$, with slightly lower variability in iRBC cultures at later time points, presumably because the abundant metabolites produced by late-stage parasites were quantified with more precision. Overall, the variability at all time points was low, demonstrating the robustness of the culture and metabolomics methods. Thus, in the following analysis, the data were averaged over replicates.

To further gauge time-dependent changes in overall metabolic activity, the average fold change in metabolite abundance $(\overline{\mathrm{FC}})$ was calculated between each pair of time points. Figure $2 \mathrm{~d}$ shows this quantity for both culture systems, where the diagonal elements are zero (much as in a correlation matrix) because the metabolite abundance at each time point is compared to itself. For uRBCs, the changes were modest (Fig. 2d, top), as reflected in the subtle changes between the early $(0-8 \mathrm{~h})$ and late time points (Fig. 2b, black clusters). In contrast, for iRBCs the changes were greater (Fig. $2 \mathrm{~d}$, bottom), as reflected in the pronounced changes between the early and late time points (Fig. 2b, red clusters).

To identify the underlying groups of metabolites that drive the changes in metabolite profiles, hierarchical clustering of all 501 metabolites was performed and metabolites that behaved differently in the two culture systems were characterized. Here, the clustering procedure grouped metabolites based on the Euclidean distance similarity of the metabolite fold-change values (see "Methods"). Figure 2e shows that most metabolites could be grouped into five distinct clusters (Clusters 1-5) based on their wide-ranging changes over time in response to parasite infection. Cluster 1 consisted mainly of metabolites that were consistently lower in $\mathrm{URBC}$ cultures than in $\mathrm{iRBC}$ cultures and higher in $\mathrm{IRBC}$ cultures than in uRBC cultures across all time points. Cluster 3 exhibited a trend opposite to Cluster 1, whereas iRBC metabolites in Clusters 4 and 5 showed marked time-dependent changes associated with IDC progression. Cluster 2 contained the largest number of metabolites, whose fold-change values were mainly of smaller magnitude and less dependent on time compared to the other clusters.

Cluster enrichment analysis (see "Methods") was used to ascertain whether specific metabolite classes could be associated with these clusters. Clusters 1 and 5 were enriched in nucleotide metabolites, Clusters 2 and 3 in lipid metabolites, and Cluster 4 in carbohydrate metabolites. This is commensurate with known global metabolic features associated with parasite-infected erythrocyte cultures during the IDC [28-30], i.e., both time-dependent and time-independent production of nucleotide metabolites in Clusters 5 and 1, respectively; consumption of carbohydrate metabolites in Cluster 4; and an increased depletion of lipids needed to create membranes for parasite progeny in Cluster 3.

\section{Metabolite changes characterizing uninfected and infected erythrocyte cultures}

This section describes the quantification of consistent, large metabolite changes between the infected and uninfected cultures-an approach that can potentially identify circulating metabolite biomarkers indicative of malaria infection. Ninety-three metabolites increased (or decreased) twofold in average abundance in iRBC cultures relative to $\mathrm{uRBC}$ cultures. Table 2 (top) shows the 15 metabolites that increased most in abundance, with fold changes ranging from $\sim 4$ (mannose-6-phosphate) to $\sim 64$ (pipecolate). These belong to Clusters 1 and 5 (Fig. 2e) and correspond to metabolites that either consistently increased in iRBC cultures (Cluster 1), or which showed a clear time-dependent increase in abundance at later time points (Cluster 5). Similarly, Table 2 (bottom) shows the 15 metabolites that decreased the most in average abundance, with fold changes ranging from $\sim 3$ ( $N^{6}$-carboxymethyllysine) to $\sim 33$ (fructose-6-phosphate). These metabolites, which all belong to Cluster 4 (Fig. 2e), were representative of a set of metabolites that consistently decreased in abundance in a stage-dependent manner with IDC progression. Additional files 4 and 5 include the average and temporal fold changes in abundance, respectively, for all 93 metabolites.

The largest overall increase in metabolite abundance occurred for pipecolate, a product of lysine catabolism. The increase in pipecolate was associated with the schizont stage (32-40 h) of the iRBC (Additional file 5), in broad agreement with a recent study [18]. Furthermore, pipecolate, which accumulates in the plasma [17] and urine $[31,32]$ of patients with severe malaria, has been suggested as a candidate clinical biomarker of malaria. Pipecolate is strongly associated with parasite-infected cultures and may be necessary for IDC progression; it is also associated with increased inflammation [33], oxidative stress [34, 35], and epilepsy [36]. The largest 
Table 2 Fold changes in metabolite levels between infected and uninfected erythrocyte cultures

\begin{tabular}{|c|c|c|c|c|}
\hline Metabolite & $\mathrm{FC}_{\mathrm{IDC}}(\mathrm{SD})^{\mathrm{a}}$ & Pathway & Mass $(\mathrm{amu})^{\mathbf{b}}$ & Clusterc \\
\hline \multicolumn{5}{|c|}{15 metabolites showing the greatest increase } \\
\hline Pipecolate & $63.9(10.1)$ & Lysine degradation & 130.1 & 5 \\
\hline Nicotinic acid mononucleotide & $33.6(3.19)$ & Nicotinate and nicotinamide metabolism & 336.0 & 5 \\
\hline Orotate & $19.8(2.74)$ & Pyrimidine metabolism & 155.0 & 1 \\
\hline Phosphoethanolamine & $13.6(1.14)$ & Phospholipid metabolism & 140.0 & 5 \\
\hline Glycerol 2-phosphate & $11.0(1.15)$ & Glycerolipid metabolism & 171.0 & 5 \\
\hline$N^{1}, N^{12}$-diacetylspermine & $7.84(1.73)$ & Polyamine metabolism & 287.2 & 1 \\
\hline $\mathrm{N}$-acetylserine & $7.71(0.36)$ & Glycine, serine, and threonine metabolism & 146.0 & 1 \\
\hline Glycerol & $7.42(0.98)$ & Glycerolipid metabolism & 91.0 & 1 \\
\hline Glycerophosphoglycerol & $5.29(0.23)$ & Glycerolipid metabolism & 245.0 & 1 \\
\hline Guanine & $4.92(0.89)$ & Purine metabolism & 152.1 & 1 \\
\hline Putrescine & $4.87(0.83)$ & Polyamine metabolism & 89.1 & 5 \\
\hline $2^{\prime}$-deoxyuridine & $4.80(0.66)$ & Pyrimidine metabolism & 227.1 & 5 \\
\hline Nicotinate ribonucleoside & $4.73(0.67)$ & Nicotinate and nicotinamide metabolism & 256.1 & 5 \\
\hline 1-stearoyl-GPG (18:0) & $4.69(0.81)$ & Lysophospholipid & 511.3 & 5 \\
\hline Mannose-6-phosphate & $4.29(1.26)$ & Fructose, mannose, and galactose metabolism & 259.0 & 5 \\
\hline \multicolumn{5}{|c|}{15 metabolites showing the greatest decrease } \\
\hline$N^{6}$-carboxymethyllysine & $0.37(0.04)$ & Advanced glycation end-product & 205.1 & 4 \\
\hline S-lactoylglutathione & $0.37(0.03)$ & Glutathione metabolism & 378.1 & 4 \\
\hline Pyrraline & $0.36(0.06)$ & Food component/plant & 255.1 & 4 \\
\hline Dihydroxyacetone phosphate & $0.36(0.03)$ & Glycolysis, gluconeogenesis, and pyruvate metabolism & 169.0 & 4 \\
\hline 3-methylcytidine & $0.31(0.04)$ & Pyrimidine metabolism & 258.1 & 4 \\
\hline 1-linoleoyl-GPC (18:2) & $0.30(0.04)$ & Lysophospholipid & 520.3 & 4 \\
\hline 2-phosphoglycerate & $0.29(0.08)$ & Glycolysis, gluconeogenesis, and pyruvate metabolism & 185.0 & 4 \\
\hline Ribitol & $0.27(0.03)$ & Pentose metabolism & 151.1 & 4 \\
\hline Gamma-glutamylglutamate & $0.27(0.03)$ & Gamma-glutamyl amino acid & 277.1 & 4 \\
\hline Isovalerylglycine & $0.26(0.05)$ & Leucine, isoleucine, and valine metabolism & 158.1 & 4 \\
\hline 1-stearoyl-GPC (18:0) & $0.25(0.04)$ & Lysophospholipid & 524.4 & 4 \\
\hline 1-oleoyl-GPC (18:1) & $0.22(0.04)$ & Lysophospholipid & 522.4 & 4 \\
\hline 1-palmitoyl-GPC (16:0) & $0.20(0.03)$ & Lysophospholipid & 496.3 & 4 \\
\hline Sedoheptulose-7-phosphate & $0.10(0.01)$ & Pentose phosphate pathway & 289.0 & 4 \\
\hline Fructose-6-phosphate & $0.03(0.00)$ & Glycolysis, gluconeogenesis, and pyruvate metabolism & 259.0 & 4 \\
\hline
\end{tabular}

amu atomic mass unit, GPC glycerophosphocholine, GPG glycerophosphoglycerol, GPI glycerophosphoinositol, GPS glycerophosphoserine, iRBC infected erythrocyte, $S D$ standard deviation, $U R B C$ uninfected erythrocyte

a Fold-change $\left(\mathrm{FC}_{\mathrm{IDC}}\right)$ values based on a comparison of the average abundance of a metabolite during the IDC in the iRBC culture relative to that in the uRBC culture

b Values provided by METABOLON@

c Cluster in Fig. 2e containing the metabolite

reduction in metabolite abundance occurred for fructose-6-phosphate, a product of glycolysis breakdown, reflecting the high glucose-consumption capacity of parasite-infected erythrocytes [29]. Within infected erythrocytes, most of the glucose $(60-70 \%)$ is incompletely oxidized to lactate and excreted [29]. High lactate dehydrogenase activity, which produces lactate from pyruvate, generates oxidized nicotinamide adenine dinucleotide $\left(\mathrm{NAD}^{+}\right)$from reduced nicotinamide adenine dinucleotide $(\mathrm{NADH})$ [37]. This process can generate nicotinic acid mononucleotide as a byproduct, which increased 33-fold in abundance (Table 2). Specifically, as $\mathrm{NAD}^{+}$increases, nicotinate-nucleotide adenylyltransferase catalyzes the conversion of $\mathrm{NAD}^{+}$into nicotinic acid mononucleotide [38], resulting in the concomitant accumulation of intraerythrocytic nicotinic acid mononucleotide.

For all metabolites present in both $\mathrm{URBC}$ and $\mathrm{iRBC}$ cultures, the observed differences in abundance represent both a RBC response to infection and a parasite component, providing a direct measurement of the intraerythrocytic environment. The largest changes in metabolite abundance occurred mostly for small molecules. Such 
molecules, which are subject to further biotransformation, may have alternative sources apart from RBC metabolism and, hence, may not be linked readily to observable plasma and urine biomarkers. Interestingly, the data in Table 2 also point to significant changes in a specific lysophospholipid (GPG 18:0), which has not been reported before.

\section{Global and temporal metabolic activity increase during the IDC}

Infected erythrocyte cultures exhibited continuous and distinct metabolic activity. Figure 3a shows the fold change in the abundance of the top 93 metabolites at the six measured time points (see Additional file 4 for their average fold changes). The magnitude of these fold changes increased from early to later time points. Figure $3 \mathrm{~b}$ shows that the number of metabolites changing by at least twofold in abundance at each time point
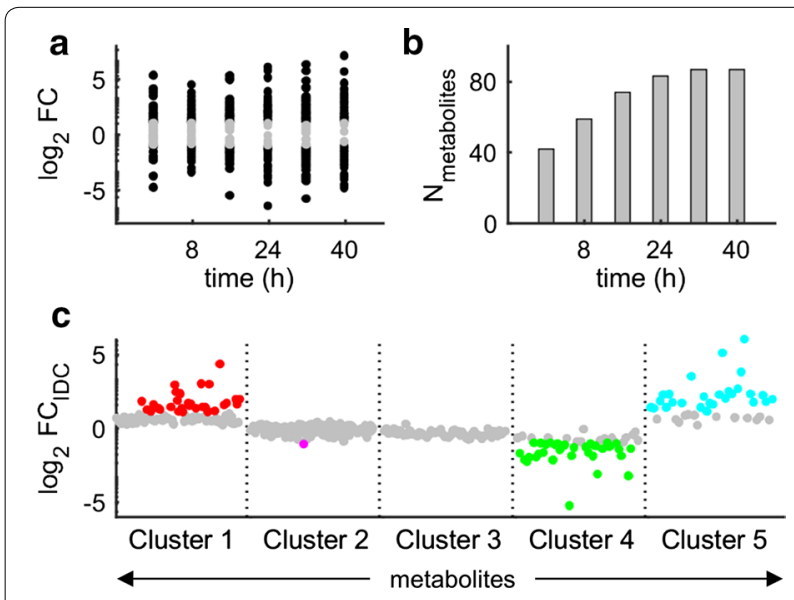

Fig. 3 Global and temporal changes in metabolite abundance during the IDC. a Temporal fold-change values in significantly altered metabolites. Here, any metabolite that changed (i.e., increased or decreased) twofold or more in abundance was considered as significantly altered. The time-specific fold change was computed as $\mathrm{m}_{\mathrm{RBC}} / \mathrm{m}_{\mathrm{URBC}}$, where $\mathrm{m}$ represents the metabolite abundance at 0,8 , $16,24,32$, or $40 \mathrm{~h}$, and $\mathrm{URBC}$ and IRBC denote $\mathrm{RRBC}$ and iRBC cultures, respectively. Fold-change values greater than or equal to two are shown in black, and those smaller than two are shown in grey. $\mathbf{b}$ The number of metabolites in $\mathbf{a}$ that changed by twofold or more at the indicated time points. The number increased monotonically with time, suggesting that pronounced metabolic changes occur during the later stages of the IDC. $\mathbf{c}$ Fold change in the average abundance of metabolites from Clusters 1-5 in Fig. 2e. The fold change in average abundance $\left(\mathrm{FC}_{\mathrm{IDC}}\right)$ was computed as $\overline{\mathrm{m}}_{\mathrm{iRBC}} / \overline{\mathrm{m}}_{\mathrm{URBC}}$, where $\bar{m}$ represents the average abundance of a metabolite ' $m$ ' averaged across all time points. Twofold changes in average abundance are shown in red (Cluster 1), magenta (Cluster 2), green (Cluster 4), or cyan (Cluster 5). Fold changes of less than two are shown in grey. Although Cluster 3 showed some temporal changes in metabolites (Fig. 2e), these disappeared when the $F C_{I D C}$ was computed (hence, all markers are grey). IDC intraerythrocytic developmental cycle, iRBC parasite-infected erythrocyte, $U R B C$ uninfected erythrocyte increased from $42(t=0 \mathrm{~h})$ to $87(t=40 \mathrm{~h})$, suggesting a parasite-induced increase in metabolic activity following infection and a growing divergence of activity between $\mathrm{uRBC}$ and iRBC cultures over the course of the IDC. Importantly, although iRBC cultures were less active in the early stages than in the later stages, they were not metabolically inactive.

The metabolites with the highest average fold-change values were concentrated in Clusters 1, 4, and 5 (Fig. 2e). Figure $3 \mathrm{c}$ shows the detailed fold change in average abundance of the metabolites from Clusters 1-5, where the marker colours correspond to those of the annotations for each cluster in Fig. 2e. A fold change of less than two is shown in grey. The average fold change $\left(\mathrm{FC}_{\mathrm{IDC}}\right)$ captured sustained alterations in metabolite abundance during the IDC and washed out any transient metabolic alterations [e.g., Clusters 2 and 3 metabolites in Fig. 2e showed a transient increase in $u R B C$ cultures or a transient decrease in iRBC cultures; however, only one (magenta) of these metabolites crossed the twofold magnitude threshold (Fig. 3c)]. Clusters 1 and 5 contained metabolites associated with nucleotide metabolism, with 31 (red) and 27 (cyan) increasing to twofold or more in abundance, consistent with the rapid rate of parasitic nucleic acid synthesis [28, 39, 40]. Cluster 4 contained metabolites associated with carbohydrate metabolism, which in malaria parasites is synonymous with the Embden-Meyerhof-Parnas pathway of glycolysis. Within this cluster, 34 metabolites (green) decreased twofold or more in abundance. This reduction in metabolite abundance is consistent with prior work showing that glucose consumption in Plasmodium parasites can increase to as much as 100-fold in iRBC cultures during the advanced stages of the IDC [29].

\section{Influence of metabolite alterations on metabolic pathways}

To identify and quantify the changes among these metabolites associated with parasite development during the IDC, pathway enrichment analysis was further performed on all metabolomic data (Fig. 4). Metabolites of the amino acid class, such as those produced by arginine-proline, aspartate, and glutathione metabolism, showed significant enrichment at intermediate and late time points, consistent with their canonical role in protein synthesis [41] and oxidative stress [42]. This enrichment was associated with haemoglobin degradation, which provides a major source of amino acids for Plasmodium parasites [41, 43]. In contrast, lysine degradation was the only amino acid pathway significantly enriched at all time points, suggesting that it continuously functions throughout the entire IDC. None of the carbohydrate or energy metabolism pathways were significantly enriched (Fig. 4). However, the pyruvate metabolism $(p \approx 0.03)$ 


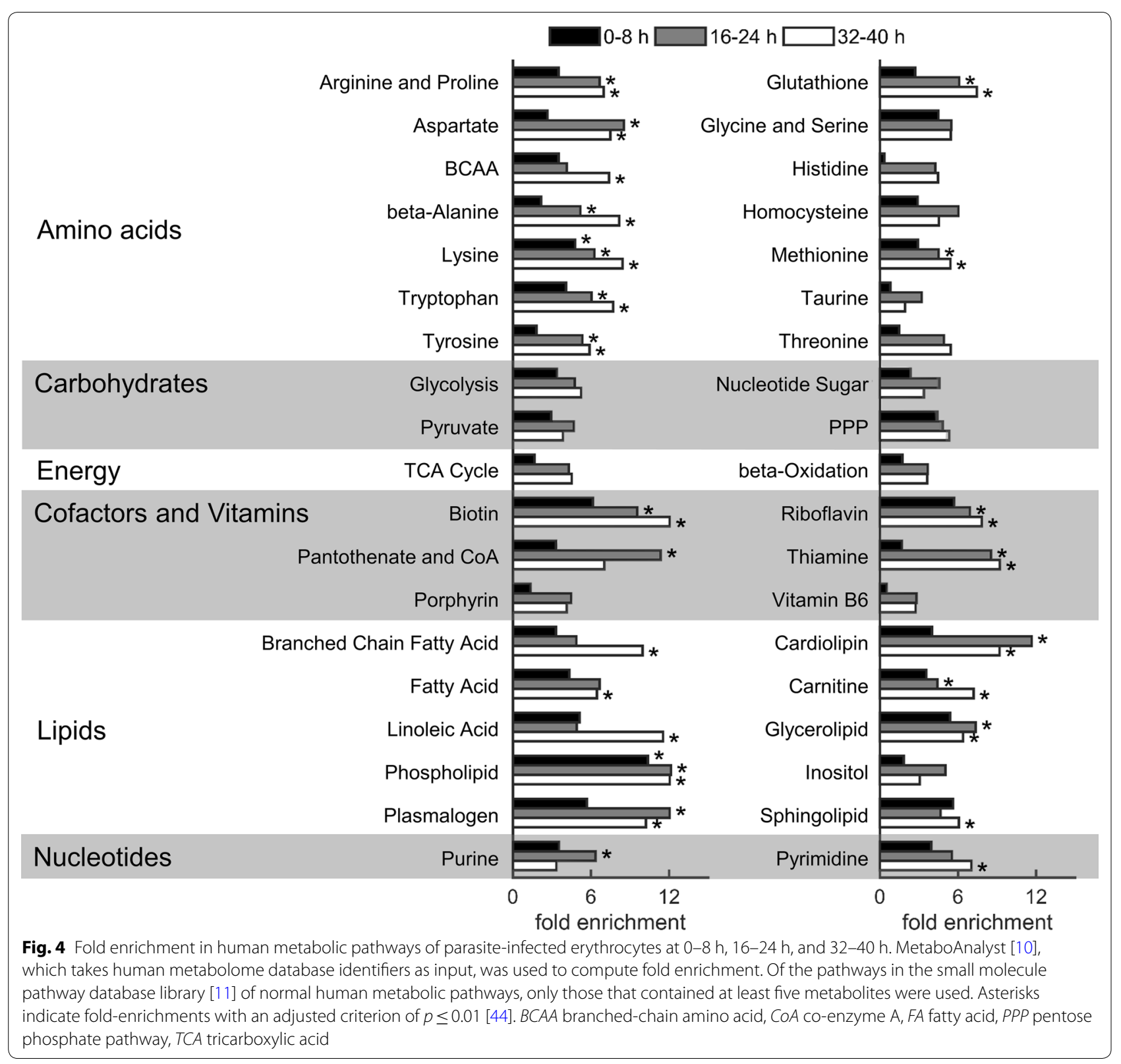

and tricarboxylic acid (TCA) cycle $(p \approx 0.01)$ pathways both showed enrichment at intermediate time points. This enrichment was associated with L-malate, which increased to 2.5-fold (Additional file 4). Neither P. falciparum parasites [45] nor RBCs have a fully-functional TCA cycle [46], which suggests that the parasite produces malate for incorporation into purine nucleotides via oxaloacetate and aspartate [47]. Among nucleotide metabolism pathways, purine showed enrichment $(\sim$ sixfold), as did pyrimidine, a metabolite synthesized de novo by $P$. falciparum [48]. Although Fig. 4 shows enrichment in several subordinate pathways of the co-factor and vitamin class, the enrichment in these pathways should not be interpreted as enrichment in any particular cofactor or vitamin metabolite, because it was more closely associated with nucleotide metabolites.

Within the lipid class, phospholipid metabolism was significantly enriched ( $>$ tenfold) at all examined time points. This enrichment was associated with metabolites involved in the syntheses of phosphatidylcholine and phosphatidylethanolamine (PtdEth), which together constitute about $75-85 \%$ of phospholipids in the parasite [49]. Dihydroxyacetone phosphate (DHAP) was also associated with enrichment in this and other lipid 
subordinate pathways, except for that of linoleic acid metabolism, which does not involve DHAP. The enrichment in linoleic acid metabolism was associated with an increased abundance of PUFAs, such as arachidonate (20:4n6), linoleate (18:2n6), docosapentaenoate (22:5n3), and docosahexaenoate (22:6n3). All of these metabolites showed increased abundance in iRBC cultures relative to uRBC cultures (Additional file 6). In mammals, docosahexaenoate $(22: 6 \mathrm{n} 3)$ and arachidonate $(20: 4 \mathrm{n} 6)$ can be synthesized from $\alpha$-linolenic acid (18:3n 3$)$ and linoleate (18:2n6), respectively [50], although synthesis of PUFAs has not previously been characterized in P. falciparum.

Overall, the enrichment in each metabolite subclass and associated metabolites showed characteristics of typical parasite development, where amino acids are used for protein synthesis, nucleotides are synthesized for DNA production, and lipids are synthesized for the development of lipid membranes. The next section describes the abundance of different phospholipid and fatty-acid metabolites in iRBC and uRBC cultures.

\section{Lipid metabolism in infected and uninfected cultures}

Figure 5a shows the fold change in abundance of phospholipids, which are characterized based on the classification system of the LIPID MAPS Structure Database [51]. GPGs and diacylglycerols (DGs) showed the largest increase. Consistent with previous reports [52, 53], a roughly fourfold increase in DGs occurred during the IDC. DGs are used to synthesize triglycerides via diacylglycerol acyltransferase, an essential enzyme during the IDC [54]. In contrast, the increased abundance in GPGs was associated with 1-palmitoyl-GPG (16:0) and 1-stearoyl-GPG (18:0), which have not previously been characterized in $P$. falciparum. These LPGs are formed when phospholipase A2 hydrolyzes phosphatidylglycerol [55], and can be reacylated by the activity of acyl-CoA:lysophosphatidylglycerol acyltransferase to form phosphatidylglycerol. A BLASThomology search of proteins homologous to human acyl-CoA:lysophosphatidylglycerol acyltransferase in the $P$. falciparum genome database [26] did not identify any candidate enzymes. This suggests that LPGs in P. falciparum may have some additional functions, such as cell proliferation [56], migration [57], cell adhesion [58], or $\mathrm{Ca}^{2+}$ signaling [59], which have been identified in mammalian cells.

Figure $5 \mathrm{~b}$ shows the distribution of fatty acids with different carbon-chain lengths in iRBC cultures relative to the $\mathrm{uRBC}$ cultures. Apart from the increase seen in a 5-carbon fatty acid, five PUFA metabolites, i.e., docosahexaenoate (22:6n3), docosapentaenoate (22:5n6), docosapentaenoate (22:5n3), mead acid (20:3n9), and eicosapentaenoate (20:5n 3$)$, increased $\sim 1.5$-fold in iRBC

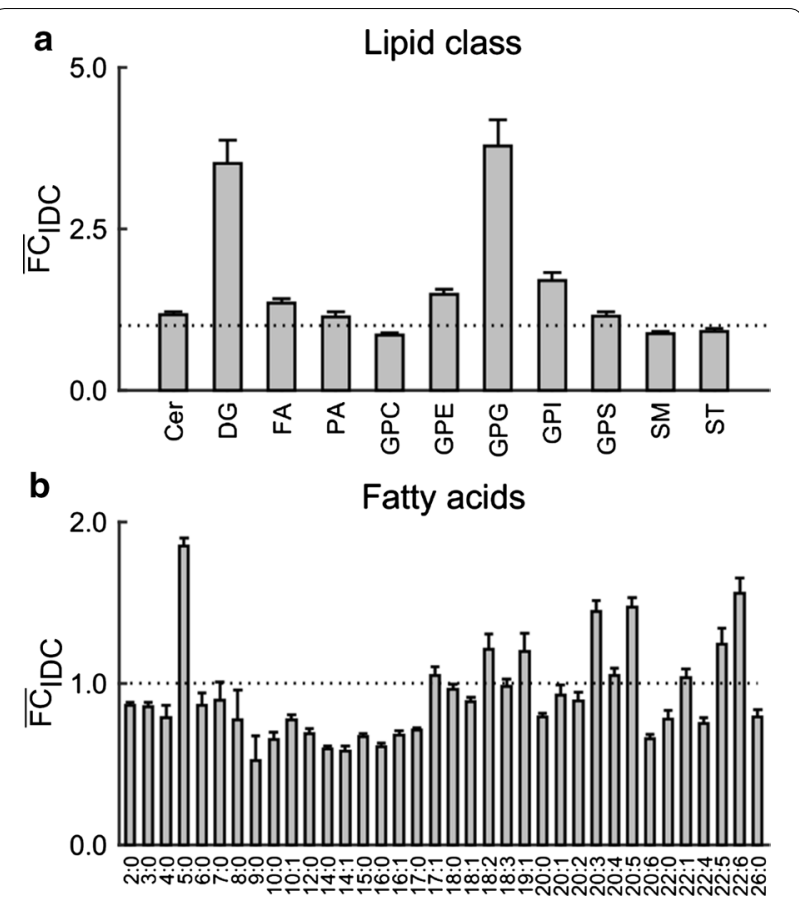

Fig. 5 Fold change in abundance of lipid and fatty acid metabolites during the intraerythrocytic developmental cycle (IDC). a Lipid metabolites were classified according to the LIPID MAPS Structure Database [51] into 13 subordinate classes. The figure shows fold changes in lipid classes that contain two or more metabolites. The fold changes were greatest for diacylglycerol (DG) and glycerophosphoglycerol (GPG) metabolites. b Fold change in abundance of fatty acids based on different carbon-chain lengths. The fold change was greatest for the 5-carbon fatty acids ( 1.8-fold in iRBC cultures relative to URBC cultures), followed by a number of 18-carbon, 20-carbon, and 22-carbon polyunsaturated fatty acids ( 1.5-fold). The $\overline{F C}_{\text {IDC }}$ was computed as the average FC (described in Fig. 3c) when a metabolite class contained more than one metabolite. Each error bar shows the standard deviation of the FCIDC of metabolites present in a metabolite class. Cer ceramide, DG diacylglycerol, FA fatty acid amide, PA glycerophosphate, GPC glycerophosphocholine, GPE glycerophosphoethanolamine, GPG glycerophosphoglycerol, GPI glycerophosphoinositol, GPS glycerophosphoserine, SM phosphosphingolipid, ST sterol

cultures relative to $\mathrm{uRBC}$ cultures (see also Additional file 7: Sheet 2). These PUFAs cannot be synthesized in mature erythrocytes as they lack an essential enzyme necessary for fatty acid biosynthesis [60]. In contrast, $P$. falciparum should be able to synthesize PUFAs because it possesses the necessary elongases and desaturases [61, 62]. PUFAs are precursors of eicosanoids, which have immunosuppressive roles [63]. P. falciparum, under in vitro conditions, can produce eicosanoids when supplemented with a PUFA [64]. Eicosanoids also mediate fever and inflammation, and have numerous other functions [65]. These data show that the parasites, under in vitro conditions, can produce precursors that mediate 
inflammatory and immunosuppressive responses in an infected human host.

The lipids quantified here were further compared with those obtained from the D2 strain of $P$. falciparum in a lipidomics study by Gulati et al. [53], in which parasites were isolated from infected erythrocytes by saponin treatment prior to LC-MS measurements and a total of 304 lipid metabolites were measured during the IDC. Most of these lipid metabolites (239 of 304) had a carbon-chain length greater than 26 , which was the maximum length in this study. In the current study, 17 (out of 65 possible) metabolites belonged to seven lipid subclasses. Following Gulati et al. [53], the abundance of a given lipid metabolite was normalized by the total lipid abundance at each time point, and then the Gulati et al. data were re-normalized to the 17 metabolites to make the datasets comparable. Table 3 lists the subclasses and their average fractions during the IDC as measured by both studies. Additional file 8 includes detailed comparisons of individual metabolites within each subclass. In agreement with the study by Gulati et al. [53], sphingomyelins - the lipid class with the third highest abundance levels in P. falciparum [53]-showed the highest abundance during the IDC (this study: $93.7 \%$ vs. Gulati et al.: 96.4\%). Notably, the abundance fraction of the lyso phosphatidylinositol (PtdIns) class was lower in the current study than in the study by Gulati et al. [53]. Given the difference in the study design (purified parasites vs. co-culture), this discrepancy suggests that lyso PtdIns metabolites are primarily synthesized by $P$. falciparum. For other lipid subclasses, there was general agreement between the studies as their average fractions during the IDC were of the same order of magnitude. Furthermore, the novel lipid classes of dihydrosphingomyelin,

Table 3 Lipid metabolites quantified in this study and Gulati et al. [53]

\begin{tabular}{llll}
\hline Lipid subclass & $\mathbf{N}_{\text {metabolites }}$ & \multicolumn{2}{l}{$\begin{array}{l}\text { Percentage of each lipid } \\
\text { subclass, } \boldsymbol{f}_{\text {IDC }}(\boldsymbol{\sigma})\end{array}$} \\
\cline { 3 - 4 } & & This study & Gulati et al. \\
\hline Sphingomyelin & 4 & $93.7(0.61)$ & $96.4(0.50)$ \\
dhSM & 2 & $4.19(0.19)$ & $1.36(0.08)$ \\
Ceramide & 2 & $0.64(0.04)$ & $0.37(0.11)$ \\
LacCer & 1 & $0.07(0.01)$ & $0.06(0.02)$ \\
Lyso PtdCho & 3 & $1.10(0.82)$ & $0.91(0.19)$ \\
Lyso PtdEth & 3 & $0.31(0.03)$ & $0.73(0.23)$ \\
Lyso Ptdlns & 2 & $0.02(0.01)$ & $0.17(0.06)$ \\
\hline
\end{tabular}

$N_{\text {metabolites }}$ number of metabolites within each subclass, $f_{\mathrm{IDC}}$ average percentage of a subclass synthesized during the intraerythrocytic developmental cycle (IDC), $\sigma$ standard deviation, dhSM dihydrosphingomyelin, LacCer lactosylceramide, PtdCho phosphatidylcholine, PtdEth phosphatidylethanolamine, Ptdlns phosphatidylinositol lyso PtdEth, and lyso PtdIns characterized by Gulati et al. [53] were also present in the current study. The final section also provides details on the quantification of a novel lysophosphatidylglycerol subclass, which was uniquely present in iRBC cultures.

\section{Discussion}

Metabolic changes that characterize parasite development The previous section described analyses of metabolic changes in lipid metabolism, which are associated with processes of the parasite that can modulate the host immune system $[64,66]$. This section considers metabolic changes in $\mathrm{iRBC}$ and $\mathrm{uRBC}$ cultures that capture the development of the parasite during the IDC. Figure 6a shows three key metabolites of glucose metabolism in $\mathrm{uRBC}$ and $\mathrm{iRBC}$ cultures. Glucose remained stable in $\mathrm{uRBC}$ cultures, whereas it decreased roughly linearly in iRBC cultures [29], ultimately becoming exhausted at the end of the IDC. Concurrently with the $\sim$ eightfold decrease of glucose in iRBC cultures at the 40-h time point relative to the 0 - $\mathrm{h}$ time point, there was a $\sim$ fivefold increase in lactate. These observations suggest that approximately $60 \%$ of the consumed glucose is oxidized to lactate in $\mathrm{iRBC}$ cultures, which is within the expected values $(60-70 \%)$ reported in the literature [29].

Phosphoenolpyruvate (PEP), a glycolysis intermediate, also showed higher abundance in iRBC cultures relative to $\mathrm{URBC}$ cultures early in the IDC $(0-16 \mathrm{~h})$, but only half the abundance in uRBC cultures later in the IDC (24$40 \mathrm{~h})$. Plasmodium falciparum converts PEP into products, such as pyruvate [67] and oxaloacetate [68], which are important metabolites for pathways including the TCA cycle. The increased utilization of PEP at later time points suggests an increased use of TCA cycle reactions late in the IDC.

There was significant enrichment in phospholipid metabolites (Fig. 4) associated with the synthesis of PtdCho and PtdEth, which comprise up to 50\% and 45\%, respectively, of the total phospholipid content in purified parasites [49]. Figure 6b shows temporal variations in phosphocholine (PCho) and phosphoethanolamine (PEth), which are precursors of PtdCho and PtdEth, respectively, in the Kennedy pathway [71]. Both metabolites exhibited the highest abundance in iRBC cultures towards the end of the IDC, commensurate with the increased demand for membrane lipids in the developing merozoites. P. falciparum can also salvage host lyso PtdCho to synthesize PtdEth [69]. Consistent with these findings, lyso PtdCho decreased $\sim$ eightfold in iRBC cultures at the 40-h time point relative to the 0 - $\mathrm{h}$ time point.

The parasite also needs to synthesize purine and pyrimidine nucleotides for DNA synthesis. It does this by salvaging purine nucleotides from the host erythrocyte 


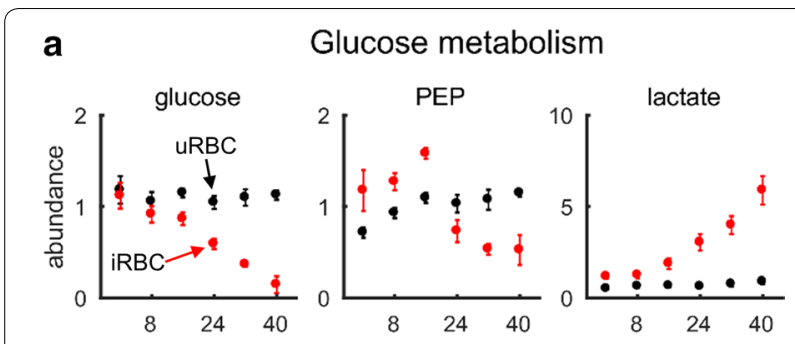

b Phospholipid metabolism

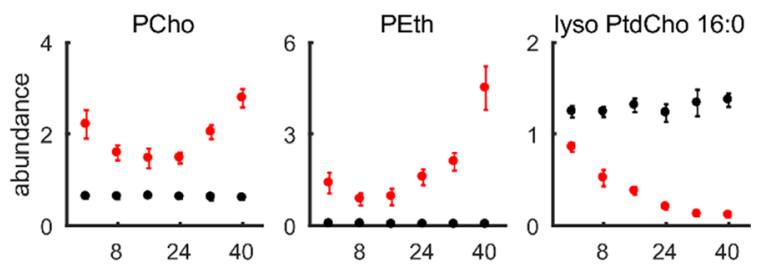

C Pyrimidine metabolism

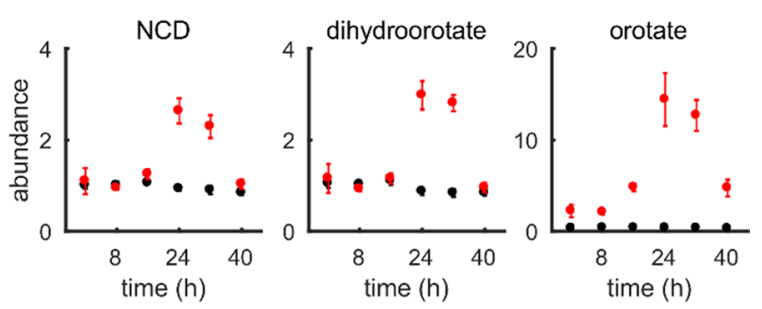

Fig. 6 Normalized abundance of important metabolites of glucose, phospholipid, and pyrimidine metabolism in uninfected (URBC) and parasite-infected erythrocyte (iRBC) cultures. a Abundance of glucose, phosphoenolpyruvate (PEP), and lactate during the intraerythrocytic developmental cycle (IDC). Glucose decreased in iRBC cultures, whereas it was stable in $U R B C$ cultures. The increase in lactate was commensurate with glucose consumption, indicating active parasite metabolism. b Abundance of phosphocholine (PCho), phosphoethanolamine (PEth), and lyso phosphatidylcholine (PtdCho) 16:0 during the IDC. PCho and PEth are precursors of PtdCho and phosphatidylethanolamine, respectively, which account for 75\% to $85 \%$ of parasite phospholipids [49]. In addition to PEth, the parasite also utilizes lyso PtdCho to synthesize PtdCho [69], which also decreased over time in iRBC cultures. c Parasites synthesize $\mathrm{N}$-carbamoyl-L-aspartate (NCD) in the first step, dihydroorotate in the second step, and orotate in the third step of de novo pyrimidine synthesis [27]. These metabolites increased in the iRBC cultures, consistent with the synthesis of parasite DNA [70]

[72], and synthesizing pyrimidine nucleotides de novo [48]. Figure 6c depicts the temporal profiles of three pyrimidine metabolites, $N$-carbamoyl-L-aspartate, dihydroorotate, and orotate, which are synthesized in the first, second, and third steps of pyrimidine metabolism, respectively [27]. All three metabolites from iRBC cultures showed a temporal pattern consistent with Plasmodium DNA synthesis [70]. In contrast, these metabolites were stable in $\mathrm{URBC}$ cultures because pyrimidine metabolites only exist in small concentrations in human

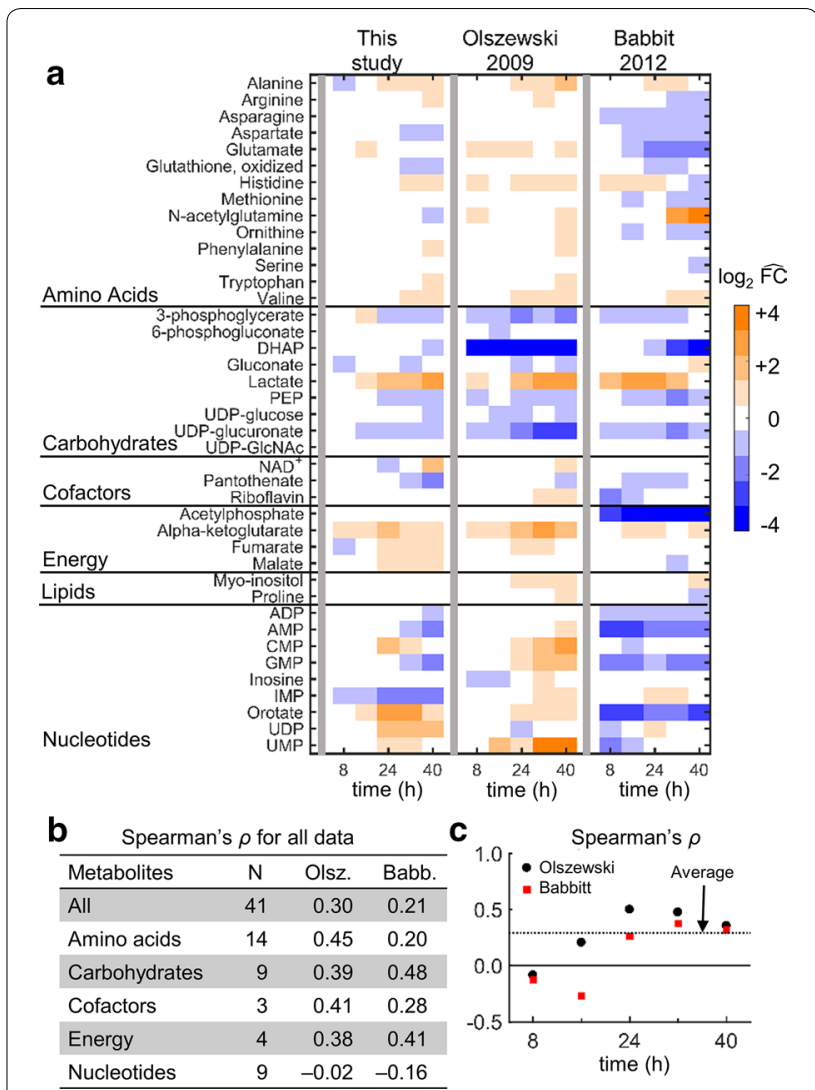

Fig. 7 Metabolite and metabolic pathway concordance between this study and studies by Olszewski et al. [4] and Babbitt et al. [8]. a Temporal profiles of metabolite abundance quantified during the intraerythrocytic developmental cycle in the three studies. Metabolite abundances were normalized by their value at $\mathrm{t}=0 \mathrm{~h}$ (grey vertical bar) to allow comparison across studies. Metabolites are grouped by metabolite class. b Spearman's $\rho$ computed for metabolites quantified in all three studies $(\mathrm{N}=41)$ and within each metabolite class. The correlation for the lipid class, which contained only two metabolites, was not computed. c Spearman's $\rho$ computed for all metabolites at 8, 16, 24, 32, and $40 \mathrm{~h}$. In comparisons with both studies, the correlation was near zero at the 8 -h time point, i.e., when parasite metabolism is least active [73]. The dotted line shows the average ( 0.3) of the correlations at each time point for both studies. ADP adenosine diphosphate, AMP adenosine monophosphate, CMP cytidine monophosphate, DHAP dihydroxyacetone phosphate,

$\widehat{F C}$ metabolite abundance normalized with respect to $t=0 \mathrm{~h}$, GMP guanosine monophosphate, IMP inosine monophosphate, $N A D^{+}$nicotinamide adenine dinucleotide (oxidized), PEP phosphoenolpyruvate, UDP uridine diphosphate, UMP uridine monophosphate

erythrocytes [48]. Taken together, the pattern of metabolic changes in the iRBC shown in Fig. 6 reflect some processes necessary for parasite development during the IDC.

Next, the results of the current study were compared with metabolite changes in iRBC cultures measured in two previous independent studies. Figure 7a shows the 
abundance of metabolites (grouped by class) during the IDC. Carbohydrate metabolites, such as lactate and PEP, showed temporal variation in abundance similar to that reported by Olszewski et al. [4] and Babbitt et al. [8]. To quantify the degree of similarity between this study and each of these two studies, Spearman's coefficient $(\rho)$ was computed for metabolites belonging to each of the five different classes (Fig. 7b). The correlation between the data in this study and Olszewski's data was highest for amino acid metabolites $(0.45 ; p \leq 0.01)$. In contrast, the same correlation between the data in this study and Babbitt's data was only $0.21(p=0.06)$. To quantify the correlation between the three studies over the course of the IDC, Spearman's $\rho$ was computed between the results of this study and those of the other two studies at each time point. The correlations were lowest early in the IDC and increased at later time points. Ring-stage parasites are less metabolically active during early stages of IDC [73] and, hence, the low correlations observed at the 8-h time point could be attributed to experimental differences between the three studies rather than differences in parasite metabolism. In contrast, parasite metabolic activity is high during the intermediate and late stages of the IDC [73], which presumably results in more robust metabolite measurements and better correlations with the two studies at the 24-h time point and beyond.

\section{Metabolites uniquely associated with parasite infection}

To identify infection-specific metabolite alterations at the early $(0-8 \mathrm{~h})$, intermediate $(16-24 \mathrm{~h})$, and late (32$40 \mathrm{~h}$ ) stages of the IDC, the variation in abundance levels was examined as a function of the infection status of the culture and time. Separate $2 \times 2$ two-way ANOVAs conducted for each of the 501 metabolites at each stage (Additional file 9), with time point (0 and $8 \mathrm{~h}, 16$ and $24 \mathrm{~h}$, or 32 and $40 \mathrm{~h}$ ) and infection status (infected and uninfected) as the between-subject factors, revealed that 42, 107, and 36 metabolites showed an infection-specific change at the early, intermediate, and late stages, respectively, as assessed by the significant interaction between time point and infection status $\left(\mathrm{F}_{1,12}\right.$ values $\geq 4.78$, $\forall p \leq 0.05 ; q<0.10$ ).

Figure 8 shows the percentages of these significantly altered metabolites that were associated with one of the five major metabolic pathways or a pathway category designated "Other" (for metabolites that did not belong to any of the major pathways). Overall, there was a relative shift from lipid to amino acid metabolism during the progression of the IDC.

At the earliest time points $(0-8 \mathrm{~h})$, significant changes occurred most frequently for metabolites belonging to lipid metabolism pathways (16 of 42: 38\%), six of which were categorized as sphingolipid metabolites. At the intermediate time points (16-24 h), the absolute number and relative fraction of lipids decreased (33 of 107:31\%) and the relative contribution of amino acid metabolism increased (33 of 107: 31\%). The decrease in lipid metabolism was also associated with a change from sphingolipid to lysophospholipid metabolism, which included metabolites that appeared uniquely in iRBC cultures, such as 1-palmitoylGPG (16:0) and 1-stearoyl-GPG (18:0) (Table 4). Both of these, which belong to the LPG class, increased more than threefold in abundance during the IDC, suggesting that they are functionally important for parasite metabolism. In mammalian cells, LPGs are known to increase intracellu$\operatorname{lar} \mathrm{Ca}^{2+}[74,75]$, although the receptor that mediates this increase is not known [76].

Late in the IDC (32-40 h), the fraction of lipid metabolites remained similar to that at $16-24 \mathrm{~h}$, although it now consisted of more phospholipids. In particular, two metabolites that also markedly increased in abundance in iRBC cultures relative to uRBC cultures (Additional file 6)
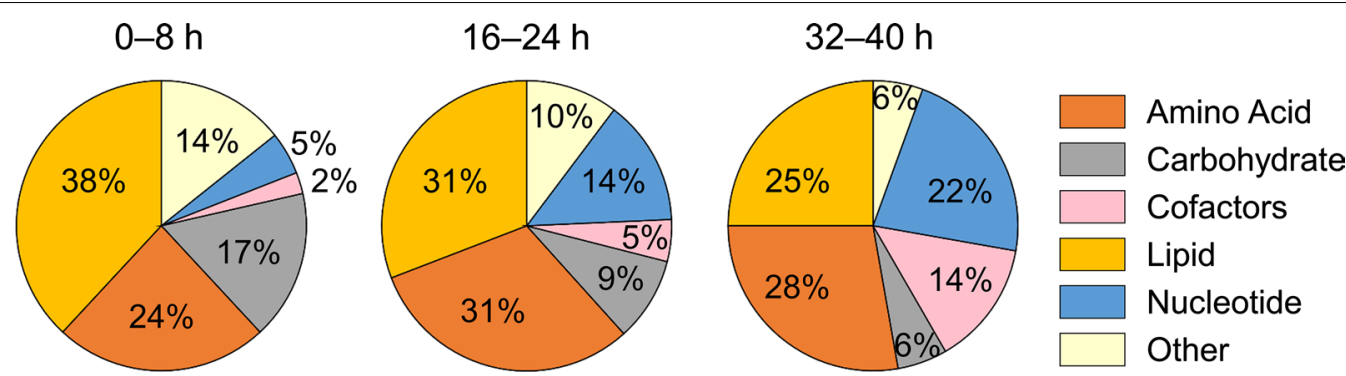

Fig. 8 Distribution of significantly altered metabolites among major metabolic pathways at 0-8 h, 16-24 h, and 32-40 h. Significantly altered metabolites $(p \leq 0.05 ; q<0.10$ ) were identified by performing a two-way analysis of variance on the metabolomic data from the cell fractions of uninfected and infected cultures at the indicated time points. At the earliest time points $(0-8 \mathrm{~h})$ most of the significantly altered metabolites belonged to the lipid class, but at later time points (16-24 h and 32-40 h) both amino acid and lipid class metabolites were equally perturbed, commensurate with the stage-dependent development of parasite metabolism. The pathway labelled "Cofactors" corresponds to metabolites that belong to cofactor and vitamin metabolism. The pathway labelled "Other" includes metabolites that do not belong to any of the major pathways 
Table 4 Metabolites uniquely detected in iRBC cultures and present at all the time points

\begin{tabular}{|c|c|c|c|}
\hline Metabolite & $F C_{I D C}(S D)^{a}$ & Pathway & Mass (amu) ${ }^{d}$ \\
\hline \multicolumn{4}{|l|}{ Amino acid metabolism } \\
\hline Putrescine & $4.87(0.83)$ & Polyamine & 89.1 \\
\hline$N^{4}$-acetylspermidine & $2.15(0.20)$ & Polyamine & 188.2 \\
\hline \multicolumn{4}{|l|}{ Cofactor and vitamin metabolism } \\
\hline Nicotinate ribonucleoside & $4.73(0.67)$ & Nicotinate and Nicotinamide & 256.1 \\
\hline Nicotinate adenine dinucleotide & $1.68(0.16)$ & Nicotinate and Nicotinamide & 663.1 \\
\hline \multicolumn{4}{|l|}{ Lipid metabolism } \\
\hline 1-stearoyl-GPG (18:0) & $4.69(0.81)$ & Lysophospholipid & 511.3 \\
\hline 1-palmitoyl-GPG (16:0) $)^{b}$ & $3.51(0.50)$ & Lysophospholipid & 483.3 \\
\hline Inositol 1-phosphate & $2.58(0.23)$ & Inositol & 259.0 \\
\hline 1-oleoyl-GPI $(18: 1)^{b}$ & $2.46(0.27)$ & Lysophospholipid & 597.3 \\
\hline Stearoyl-arachidonoyl-glycerol $(18: 0 / 20: 4)^{b, c}$ & $1.68(0.11)$ & Diacylglycerol & 662.6 \\
\hline Palmitoyl-linolenoyl-glycerol (16:0/18:3) ${ }^{b}$ & $1.59(0.20)$ & Diacylglycerol & 608.5 \\
\hline 1-arachidonoyl-GPI (20:4) & $1.51(0.12)$ & Lysophospholipid & 619.3 \\
\hline 1-oleoyl-GPS (18:1) & $1.49(0.19)$ & Lysophospholipid & 522.3 \\
\hline \multicolumn{4}{|l|}{ Nucleotide metabolism } \\
\hline Thymidine & $3.35(0.48)$ & Pyrimidine & 241.1 \\
\hline 2'-O-methylcytidine & $3.25(0.47)$ & Pyrimidine & 258.1 \\
\hline Guanosine & $2.45(0.41)$ & Purine & 284.1 \\
\hline Pseudouridine & $1.55(0.18)$ & Pyrimidine & 245.1 \\
\hline Uridine $5^{\prime}$-diphosphate & $1.42(0.20)$ & Pyrimidine & 403.0 \\
\hline 5-methyluridine & $1.29(0.19)$ & Pyrimidine & 257.1 \\
\hline \multicolumn{4}{|l|}{ Peptide } \\
\hline Histidylalanine & $1.11(0.11)$ & Dipeptide & 227.1 \\
\hline \multicolumn{4}{|c|}{ amu atomic mass unit, GPG glycerophosphoglycerol, GPI glycerophosphoinositol, GPS glycerophosphoserine, SD standard deviation } \\
\hline \multicolumn{4}{|c|}{ a Fold-change $\left(F C_{I D C}\right)$ values based on average abundance of a metabolite during the IDC in iRBC relative to the uRBC cultures } \\
\hline \multicolumn{4}{|c|}{ b Metabolite identified based on $\mathrm{m} / \mathrm{z}$ ratio alone with no external standard for validation } \\
\hline \multicolumn{4}{|c|}{${ }^{c} \mathrm{~m} / \mathrm{z}$ ratio appeared twice in the same platform, as it is a structural isomer of another compound in the METABOLON@ spectral library } \\
\hline d Values provided by METABOLON@ & & & \\
\hline
\end{tabular}

were part of this set (i.e., PCho and PEth). These metabolites also appeared in the pathway enrichment analysis. Of these, PEth showed the highest abundance [ 20-fold within this group (Additional file 5) and $~ 14$-fold during the IDC (Table 2)].

The ANOVA analysis suggests a dynamic progression of metabolic activity in iRBCs, commensurate with highly regulated processes of parasite development and the shifting demands placed on parasite metabolism. This is reflected in changes in lipid metabolism that are apparently correlated with the parasite stage-a shifting emphasis from sphingolipids and lysophospholipids involved in cell signaling, to phospholipid metabolism related to de novo synthesis and the accumulation of infection-specific membrane components.

Table 4 lists the metabolites that were uniquely detected in iRBC cultures at all time points in all four replicates. The abundance of nicotinate ribonucleoside (NR) increased fivefold in the iRBC cultures. During the IDC,
NR increases up to $\sim 15$-fold in the extra-cellular medium of iRBC cultures, but is undetectable in uRBC cultures [18], confirming the observation that NR was specific to the iRBC cultures. P. falciparum encodes an enzyme that spontaneously synthesizes NR from nicotinate mononucleotide $[27,38]$. Interestingly, nicotinate mononucleotide increased $\sim 34$-fold in iRBC cultures (Table 2). These results suggest that excessive glucose utilization in the iRBC cultures can cause accumulation of NR, which is then excreted to the extra-cellular medium [18]. As discussed above, nicotinate mononucleotide (Table 2) was associated with excessive glucose utilization. Therefore, NR has the potential to be a P. falciparum-specific blood marker of malaria infection, because these parasites are voracious consumers of glucose [29] and can cause hypoglycaemia in infected hosts [77, 78]. Additional file 10 includes a complete list of metabolites detected in iRBC and $\mathrm{uRBC}$ cultures, along with their raw counts during the IDC. 


\section{Conclusions}

High-resolution metabolomics was employed to quantify metabolic alterations in uninfected and parasite-infected erythrocyte cultures at multiple time points during the IDC. Analyses of the collected data quantified approximately fivefold more metabolites than previous studies of similar scope. Detailed analyses comparing infected and uninfected cultures, coupled with the time-course data, showed overall agreement with previous studies of $P$. falciparum blood-stage infection, but also revealed new insights. In particular, a new phospholipid class of LPG metabolites present only in parasite-infected cultures was identified. Although these metabolites modulate $\mathrm{Ca}^{2+}$ signaling in mammalian cells [59], their functional role in $P$. falciparum biology is unknown.

The relative abundance of these polyunsaturated fatty acids in the iRBC cultures increased. These metabolites are precursors for synthesizing eicosanoid-signaling molecules [79], which when released into the host blood plasma can modulate fever and inflammation under in vivo conditions [63]. They are also known to promote gametocytogenesis [80] and, thus, may play a role during the blood-stage development of $P$. falciparum.

The improved characterization and quantification of lipid metabolites reveals highly dynamic usage of phospholipids during the IDC. Specifically, the results suggest that sphingolipid and lysophospholipid metabolites are significantly utilized at early and intermediate stages of the IDC, whereas phospholipid metabolites dominate during the late stages. Overall, the metabolomic data presented here provide an opportunity for targeted discovery of metabolic functions and biology in P. falciparum.

\section{Supplementary information}

Supplementary information accompanies this paper at https://doi. org/10.1186/s12936-020-03174-z.

Additional file 1. List of metabolites not annotated in KEGG and HMDB database.

Additional file 2. List of metabolites annotated in Plasmodium metabolite database.

Additional file 3. List of metabolites annotated in Malaria Parasite Metabolic Pathways database.

Additional file 4. Metabolites that have greater than twofold change in average abundance during the IDC.

Additional file 5. Fold change in abundance of metabolites listed in Additional file 4 at 0, 8, 16, 24, 32, and $40 \mathrm{~h}$ time point.

Additional file 6 . Fold change in average abundance of all metabolites detected in $\mathrm{URBC}$ and $\mathrm{IRBC}$ cultures during the IDC.

Additional file 7. Fold change in average abundance of lipids (Sheet 1) and fatty acids (Sheet 2) related to Fig. 5.

Additional file 8. Comparison of lipid metabolites from this study with Gulati et al. [53].
Additional file 9. Results of two-way ANOVA performed on data from URBC and iRBC cultures obtained at 0-8 h (Sheet 1), 16-24 h (Sheet 2), and 32-40 h (Sheet 3).

Additional file 10. Raw metabolomic data from $\mathrm{URBC}$ and $\mathrm{RBC}$ cultures obtained in quadruplicate at $0,8,16,24,32$, and $40 \mathrm{~h}$ time point.

\section{Abbreviations}

ADP: Adenosine diphosphate; AMP: Adenosine monophosphate; amu: Atomic mass unit; ANOVA: Analysis of variance; BCAA: Branched-chain amino acid; CMP: Cytidine monophosphate; CoA: Co-enzyme A; DG: Diacylglycerol; DHAP: Dihydroxyacetone phosphate; dhSM: Dihydrosphingomyelin; ESI: Electrospray ionization; FA: Fatty acid; GMP: Guanosine monophosphate; GPC: Glycerophosphocholine; GPE: Glycerophosphoethanolamine; GPG: Glycerophosphoglycerol; GPI: Glycerophosphoinositol; GPS: Glycerophosphoserine; HCA: Hierarchical clustering analysis; HILIC: Hydrophilic-interaction chromatography; HMDB: Human metabolome database; IDC: Intraerythrocytic developmental cycle; IMP: Inosine monophosphate; iRBC: Parasite-infected erythrocyte culture; KEGG: Kyoto encyclopedia of genes and genomes; LacCer: Lactosylceramide; LPG: Lysophosphatidylglycerol; MACS: Magnetically activated cell sorting; $\mathrm{NAD}^{+}$: Nicotinamide adenine dinucleotide (oxidized); NADH: Nicotinamide adenine dinucleotide (reduced); NCD: N-carbamoyl-L-aspartate; NR: Nicotinate ribonucleoside; PC1: First principal component; PC2: Second principal component; PC3: Third principal component; PCA: Principal component analysis; PCho: Phosphocholine; PEP: Phosphoenolpyruvate; PEth: Phosphoethanolamine; PPP: Pentose phosphate pathway; PtdCho: Phosphatidylcholine; PtdEth: Phosphatidylethanolamine; Ptdlns: Phosphatidylinositol; PUFA: Polyunsaturated fatty acid; QSEA: Quantitative pathway enrichment analysis; RP: Reverse phase; RPMI: Roswell Park Memorial Institute; SD: Standard deviation; SMPDB: Small molecule pathway database; TCA: Tricarboxylic acid; UDP: Uridine diphosphate; UMP: Uridine monophosphate; UPLC: Ultrahigh-performance liquid chromatography; uRBC: Uninfected erythrocyte culture; WBC: White blood cell; WHO: World Health Organization.

\section{Acknowledgements}

The authors thank Dr. Tatsuya Oyama for his comments on a previous version of this manuscript. The authors would also like to thank Dr. Hagai Ginsburg for providing the list of metabolites available in the Malaria Parasite Metabolic Pathways database. The opinions and assertions contained herein are the private views of the authors and are not to be construed as official or as reflecting the views of the U.S. Army, the U.S. Department of Defense, or The Henry M. Jackson Foundation for the Advancement of Military Medicine, Inc. (HJF). This manuscript has been approved for public release with unlimited distribution.

\section{Authors' contributions}

SGT, JR, and AW designed the research; SGT performed the research; RS and STP collected the data; SGT analysed the data; SGT and AW wrote the manuscript; all authors edited the manuscript; and JR edited the final version of the manuscript. All authors read and approved the final manuscript.

\section{Funding}

This work was primarily funded by the Network Science Initiative of the U.S. Army Medical Research and Development Command, Ft. Detrick, Maryland (Award W81XWH-15-C-0061; STP). This work also leveraged support by the National Institutes of Health R01 Al065853 (STP), the Johns Hopkins Malaria Research Institute, the Bloomberg Family Foundation, and the NIH National Center for Research Resources UL1 RR025005.

\section{Availability of data and materials}

All data generated or analysed during this study are included in this published article and its additional files.

\section{Ethics approval and consent to participate}

Not applicable.

\section{Consent for publication}

Not applicable. 


\section{Competing interests}

The authors declare that they have no competing interests.

\section{Author details}

${ }^{1}$ Department of Defense Biotechnology High Performance Computing Software Applications Institute, Telemedicine and Advanced Technology Research Center, U.S. Army Medical Research and Development Command, Ft. Detrick, MD, USA. ${ }^{2}$ The Henry M. Jackson Foundation for the Advancement of Military Medicine, Inc. (HJF), Bethesda, MD, USA. ${ }^{3}$ Department of Molecular Microbiology and Immunology, Johns Hopkins University, Baltimore, MD, USA.

Received: 27 November 2019 Accepted: 20 February 2020

Published online: 27 February 2020

\section{References}

1. WHO. World malaria report 2018. Geneva: World Health Organization; 2018.

2. Bozdech Z, Llinas M, Pulliam BL, Wong ED, Zhu J, DeRisi JL. The transcriptome of the intraerythrocytic developmental cycle of Plasmodium falciparum. PLoS Biol. 2003;1:E5.

3. Counihan NA, Chisholm SA, Bullen HE, Srivastava A, Sanders PR, Jonsdottir TK, et al. Plasmodium falciparum parasites deploy RhopH2 into the host erythrocyte to obtain nutrients, grow and replicate. Elife. 2017;6:e23217.

4. Olszewski KL, Morrisey JM, Wilinski D, Burns JM, Vaidya AB, Rabinowitz JD, et al. Host-parasite interactions revealed by Plasmodium falciparum metabolomics. Cell Host Microbe. 2009;5:191-9.

5. Cobbold SA, McConville MJ. Determining the mode of action of antimalarial drugs using time-resolved LC-MS-based metabolite profiling. Methods Mol Biol. 2019;1859:225-39.

6. Creek DJ, Chua HH, Cobbold SA, Nijagal B, MacRae JI, Dickerman BK, et al. Metabolomics-based screening of the malaria box reveals both novel and established mechanisms of action. Antimicrob Agents Chemother. 2016:60:6650-63.

7. Kloehn J, Blume M, Cobbold SA, Saunders EC, Dagley MJ, McConville MJ. Using metabolomics to dissect host-parasite interactions. Curr Opin Microbiol. 2016;32:59-65.

8. Babbitt SE, Altenhofen L, Cobbold SA, Istvan ES, Fennell C, Doerig C, et al. Plasmodium falciparum responds to amino acid starvation by entering into a hibernatory state. Proc Natl Acad Sci USA. 2012;109:E3278-87.

9. Ben Mamoun C, Prigge ST, Vial H. Targeting the lipid metabolic pathways for the treatment of malaria. Drug Dev Res. 2010;71:44-55.

10. Chong J, Yamamoto M, Xia J. MetaboAnalystR 2.0: from raw spectra to biological insights. Metabolites. 2019;9:E57.

11. Jewison T, Su Y, Disfany FM, Liang Y, Knox C, Maciejewski A, et al. SMPDB 2.0: big improvements to the Small Molecule Pathway Database. Nucleic Acids Res. 2014;42:D478-84.

12. Kanehisa M, Goto S. KEGG: Kyoto Encyclopedia of Genes and Genomes. Nucleic Acids Res. 2000;28:27-30.

13. Biau DJ, Kerneis S, Porcher R. Statistics in brief: the importance of sample size in the planning and interpretation of medical research. Clin Orthop Relat Res. 2008;466:2282-8.

14. McDonald JH. Handbook of biological statistics. 3rd ed. Baltimore: Sparky House Publishing; 2009

15. Storey JD. A direct approach to false discovery rates. J R Stat Soc Series B Stat Methodol. 2002:64:479-98.

16. Teng R, Junankar PR, Bubb WA, Rae C, Mercier P, Kirk K. Metabolite profiling of the intraerythrocytic malaria parasite Plasmodium falciparum by ${ }^{1} \mathrm{H}$ NMR spectroscopy. NMR Biomed. 2009;22:292-302.

17. Leopold SJ, Ghose A, Allman EL, Kingston HWF, Hossain A, Dutta AK, et al. Identifying the components of acidosis in patients with severe Plasmodium falciparum malaria using metabolomics. J Infect Dis. 2019:219:1766-76.

18. Beri D, Ramdani G, Balan B, Gadara D, Poojary M, Momeux L, et al. Insights into physiological roles of unique metabolites released from Plasmodiuminfected RBCs and their potential as clinical biomarkers for malaria. Sci Rep. 2019;9:2875.

19. Salinas JL, Kissinger JC, Jones DP, Galinski MR. Metabolomics in the fight against malaria. Mem Inst Oswaldo Cruz. 2014;109:589-97.
20. Sana TR, Gordon DB, Fischer SM, Tichy SE, Kitagawa N, Lai C, et al. Global mass spectrometry based metabolomics profiling of erythrocytes infected with Plasmodium falciparum. PLOS ONE. 2013;8:e60840.

21. Cobbold SA, Chua HH, Nijagal B, Creek DJ, Ralph SA, McConville MJ. Metabolic dysregulation induced in Plasmodium falciparum by dihydroartemisinin and other front-line antimalarial drugs. J Infect Dis. 2016;213:276-86

22. Allman EL, Painter HJ, Samra J, Carrasquilla M, Llinás M. Metabolomic profiling of the malaria box reveals antimalarial target pathways. Antimicrob Agents Chemother. 2016;60:6635-49.

23. Siddiqui G, Srivastava A, Russell AS, Creek DJ. Multi-omics based identification of specific biochemical changes associated with PfKelch13mutant artemisinin-resistant Plasmodium falciparum. J Infect Dis. 2017:215:1435-44.

24. Carey MA, Covelli V, Brown A, Medlock GL, Haaren M, Cooper JG, et al. Influential parameters for the analysis of intracellular parasite metabolomics. mSphere. 2018;3:e00097-18.

25. Wishart DS, Feunang YD, Marcu A, Guo AC, Liang K, Vazquez-Fresno R, et al. HMDB 4.0: the human metabolome database for 2018. Nucleic Acids Res. 2018;46:D608-17.

26. Bahl A, Brunk B, Crabtree J, Fraunholz MJ, Gajria B, Grant GR, et al. PlasmoDB: the Plasmodium genome resource. A database integrating experimental and computational data. Nucleic Acids Res. 2003;31:212-5.

27. Ginsburg H, Tilley L. Plasmodium falciparum metabolic pathways (MPMP) project upgraded with a database of subcellular locations of gene products. Trends Parasitol. 2011;27:285-6.

28. Matthews H, Duffy CW, Merrick CJ. Checks and balances? DNA replication and the cell cycle in Plasmodium. Parasit Vectors. 2018;11:216.

29. Olszewski KL, Llinas M. Central carbon metabolism of Plasmodium parasites. Mol Biochem Parasitol. 2011;175:95-103.

30. Mitamura T, Palacpac NM. Lipid metabolism in Plasmodium falciparuminfected erythrocytes: possible new targets for malaria chemotherapy. Microbes Infect. 2003;5:545-52.

31. Sengupta A, Ghosh S, Basant A, Malusare S, Johri P, Pathak S, et al. Global host metabolic response to Plasmodium vivax infection: a ${ }^{1} \mathrm{H}$ NMR based urinary metabonomic study. Malar J. 2011;10:384.

32. Abdelrazig S, Ortori CA, Davey G, Deressa W, Mulleta D, Barrett DA, et al. A metabolomic analytical approach permits identification of urinary biomarkers for Plasmodium falciparum infection: a case-control study. Malar J. 2017;16:229.

33. Pietzner M, Kaul A, Henning AK, Kastenmuller G, Artati A, Lerch MM, et al. Comprehensive metabolic profiling of chronic low-grade inflammation among generally healthy individuals. BMC Med. 2017;15:210.

34. Krishnan N, Dickman MB, Becker DF. Proline modulates the intracellular redox environment and protects mammalian cells against oxidative stress. Free Radic Biol Med. 2008:44:671-81.

35. Dalazen GR, Terra M, Jacques CE, Coelho JG, Freitas R, Mazzola PN, et al. Pipecolic acid induces oxidative stress in vitro in cerebral cortex of young rats and the protective role of lipoic acid. Metab Brain Dis. 2014;29:175-83.

36. Wempe MF, Kumar A, Kumar V, Choi YJ, Swanson MA, Friederich MW, et al. Identification of a novel biomarker for pyridoxine-dependent epilepsy: implications for newborn screening. J Inherit Metab Dis. 2019:42:565-74.

37. Wirth JD, Boucher Jl, Jacobowitz JR, Classen S, Theobald DL. Functional and structural resilience of the active site loop in the evolution of Plasmodium lactate dehydrogenase. Biochemistry. 2018;57:6434-42.

38. Flamholz A, Noor E, Bar-Even A, Milo R. eQuilibrator-the biochemical thermodynamics calculator. Nucleic Acids Res. 2012;40:D770-5.

39. Tewari SG, Rajaram K, Schyman P, Swift R, Reifman J, Prigge ST, et al. Short-term metabolic adjustments in Plasmodium falciparum counter hypoxanthine deprivation at the expense of long-term viability. Malar J. 2019;18:86.

40. Gritzmacher CA, Reese RT. Protein and nucleic acid synthesis during synchronized growth of Plasmodium falciparum. J Bacteriol. 1984;160:1165-7.

41. Sherman IW. Amino acid metabolism and protein synthesis in malarial parasites. Bull World Health Organ. 1977;55:265-76.

42. Becker K, Rahlfs S, Nickel C, Schirmer RH. Glutathione-functions and metabolism in the malarial parasite Plasmodium falciparum. Biol Chem. 2003:384:551-66. 
43. Goldberg DE. Complex nature of malaria parasite hemoglobin degradation. Proc Natl Acad Sci USA. 2013;110:5283-4.

44. Aickin $\mathrm{M}$, Gensler $\mathrm{H}$. Adjusting for multiple testing when reporting research results: the Bonferroni vs Holm methods. Am J Public Health. 1996;86:726-8.

45. Ke H, Lewis IA, Morrisey JM, McLean KJ, Ganesan SM, Painter HJ, et al. Genetic investigation of tricarboxylic acid metabolism during the Plasmodium falciparum life cycle. Cell Rep. 2015;11:164-74.

46. van Wijk $R$, van Solinge WW. The energy-less red blood cell is lost: erythrocyte enzyme abnormalities of glycolysis. Blood. 2005;106:4034-42.

47. Wang X, Miyazaki Y, Inaoka DK, Hartuti ED, Watanabe Yl, Shiba T, et al. Identification of Plasmodium falciparum mitochondrial malate: quinone oxidoreductase inhibitors from the pathogen box. Genes. 2019;10:471.

48. Cassera MB, Zhang Y, Hazleton KZ, Schramm VL. Purine and pyrimidine pathways as targets in Plasmodium falciparum. Curr Top Med Chem. 2011;11:2103-15.

49. Vial H, Penarete D, Wein S, Caldarelli S, Fraisse L, Peyrottes S. Lipids as drug targets for malaria therapy. In: Becker K, editor. Apicomplexan parasites: molecular approaches toward targeted drug development. Singapore: Wiley-Blackwell; 2011. p. 139-40.

50. Catala A. Five decades with polyunsaturated Fatty acids: chemical synthesis, enzymatic formation, lipid peroxidation and its biological effects. J Lipids. 2013;2013:710290.

51. Fahy E, Subramaniam S, Murphy RC, Nishijima M, Raetz CR, Shimizu T, et al. Update of the LIPID MAPS comprehensive classification system for lipids. J Lipid Res. 2009;50(Suppl):S9-14.

52. Tran PN, Brown SH, Rug M, Ridgway MC, Mitchell TW, Maier AG. Changes in lipid composition during sexual development of the malaria parasite Plasmodium falciparum. Malar J. 2016;15:73.

53. Gulati S, Ekland EH, Ruggles KV, Chan RB, Jayabalasingham B, Zhou B, et al. Profiling the essential nature of lipid metabolism in asexual blood and gametocyte stages of Plasmodium falciparum. Cell Host Microbe. 2015;18:371-81.

54. Palacpac NM, Hiramine Y, Seto S, Hiramatsu R, Horii T, Mitamura T. Evidence that Plasmodium falciparum diacylglycerol acyltransferase is essential for intraerythrocytic proliferation. Biochem Biophys Res Commun. 2004;321:1062-8.

55. Hite RD, Seeds MC, Safta AM, Jacinto RB, Gyves JI, Bass DA, et al. Lysophospholipid generation and phosphatidylglycerol depletion in phospholipase $\mathrm{A}_{2}$-mediated surfactant dysfunction. Am J Physiol Lung Cell Mol Physiol. 2005;288:L618-24.

56. Ferry G, Tellier E, Try A, Gres S, Naime I, Simon MF, et al. Autotaxin is released from adipocytes, catalyzes lysophosphatidic acid synthesis, and activates preadipocyte proliferation. Up-regulated expression with adipocyte differentiation and obesity. J Biol Chem. 2003;278:18162-9.

57. Matloubian M, Lo CG, Cinamon G, Lesneski MJ, Xu Y, Brinkmann V, et al. Lymphocyte egress from thymus and peripheral lymphoid organs is dependent on S1P receptor 1. Nature. 2004;427:355-60.

58. Meyer zu Heringdorf D, Jakobs KH. Lysophospholipid receptors: signalling, pharmacology and regulation by lysophospholipid metabolism. Biochim Biophys Acta. 2007;1768:923-40.

59. Meyer Zu Heringdorf D. Lysophospholipid receptor-dependent and -independent calcium signaling. J Cell Biochem. 2004;92:937-48.

60. Pittman JG, Martin DB. Fatty acid biosynthesis in human erythrocytes: evidence in mature erythrocytes for an incomplete long chain fatty acid synthesizing system. J Clin Invest. 1966;45:165-72.

61. Mazumdar J, Striepen B. Make it or take it: fatty acid metabolism of apicomplexan parasites. Eukaryot Cell. 2007;6:1727-35.

62. Gratraud P, Huws E, Falkard B, Adjalley S, Fidock DA, Berry L, et al. Oleic acid biosynthesis in Plasmodium falciparum: characterization of the stearoyl-CoA desaturase and investigation as a potential therapeutic target. PLoS ONE. 2009;4:e6889.

63. Goodwin JS, Ceuppens J. Regulation of the immune response by prostaglandins. J Clin Immunol. 1983;3:295-315.
64. Kilunga Kubata B, Eguchi N, Urade Y, Yamashita K, Mitamura T, Tai K, et al. Plasmodium falciparum produces prostaglandins that are pyrogenic, somnogenic, and immunosuppressive substances in humans. J Exp Med. 1998;188:1197-202.

65. Funk CD. Prostaglandins and leukotrienes: advances in eicosanoid biology. Science. 2001;294:1871-5.

66. Schwarzer E, Kuhn H, Valente E, Arese P. Malaria-parasitized erythrocytes and hemozoin nonenzymatically generate large amounts of hydroxy fatty acids that inhibit monocyte functions. Blood. 2003;101:722-8.

67. Chan M, Tan DS, Sim TS. Plasmodium falciparum pyruvate kinase as a novel target for antimalarial drug-screening. Travel Med Infect Dis. 2007;5:125-31.

68. Storm J, Sethia S, Blackburn GJ, Chokkathukalam A, Watson DG, Breitling $\mathrm{R}$, et al. Phosphoenolpyruvate carboxylase identified as a key enzyme in erythrocytic Plasmodium falciparum carbon metabolism. PLoS Pathog. 2014;10:e1003876.

69. Brancucci NMB, Gerdt JP, Wang C, De Niz M, Philip N, Adapa SR, et al. Lysophosphatidylcholine regulates sexual stage differentiation in the human malaria parasite Plasmodium falciparum. Cell. 2017;171:1532-44.

70. Fang X, Reifman J, Wallqvist A. Modeling metabolism and stage-specific growth of Plasmodium falciparum HB3 during the intraerythrocytic developmental cycle. Mol BioSyst. 2014;10:2526-37.

71. Kilian N, Choi JY, Voelker DR, Ben Mamoun C. Role of phospholipid synthesis in the development and differentiation of malaria parasites in the blood. J Biol Chem. 2018;293:17308-16.

72. Downie MJ, Kirk K, Mamoun CB. Purine salvage pathways in the intraerythrocytic malaria parasite Plasmodium falciparum. Eukaryot Cell. 2008;7:1231-7.

73. Wallqvist A, Fang X, Tewari SG, Ye P, Reifman J. Metabolic host responses to malarial infection during the intraerythrocytic developmental cycle. BMC Syst Biol. 2016;10:58

74. Zhang Y, Zhang JD, Zhu MQ, Zhang M, Xu YJ, Cui L, et al. Effect of lysophosphatidylglycerol on intracellular free $\mathrm{Ca}^{2+}$ concentration in A10 vascular smooth muscle cells. Can J Physiol Pharmacol. 2017;95:1283-8.

75. Park KS, Kim MK, Im DS, Bae YS. Effect of lysophosphatidylglycerol on several signaling molecules in OVCAR-3 human ovarian cancer cells: involvement of pertussis toxin-sensitive G-protein coupled receptor. Biochem Pharmacol. 2007;73:675-81.

76. Makide K, Uwamizu A, Shinjo Y, Ishiguro J, Okutani M, Inoue A, et al. Novel lysophosphoplipid receptors: their structure and function. J Lipid Res. 2014;55:1986-95

77. White NJ, Miller KD, Marsh K, Berry CD, Turner RC, Williamson DH, et al. Hypoglycaemia in African children with severe malaria. Lancet. 1987;1:708-11.

78. White NJ, Warrell DA, Chanthavanich P, Looareesuwan S, Warrell MJ, Krishna $\mathrm{S}$, et al. Severe hypoglycemia and hyperinsulinemia in falciparum malaria. N Engl J Med. 1983;309:61-6.

79. Uttaro AD. Biosynthesis of polyunsaturated fatty acids in lower eukaryotes. IUBMB Life. 2006:58:563-71.

80. Tanaka TQ, Tokuoka SM, Nakatani D, Hamano F, Kawazu SI, Wellems TE, et al. Polyunsaturated fatty acids promote Plasmodium falciparum gametocytogenesis. Biol Open. 2019;8:bio042259.

\section{Publisher's Note}

Springer Nature remains neutral with regard to jurisdictional claims in published maps and institutional affiliations. 